\title{
Influence of atmospheric conditions on the power production of utility-scale wind turbines in yaw misalignment $\odot \odot$
}

Cite as: J. Renewable Sustainable Energy 12, 063307 (2020); https://doi.org/10.1063/5.0023746 Submitted: 01 August 2020 . Accepted: 19 November 2020 . Published Online: 21 December 2020

(D) Michael F. Howland, Carlos Moral González, Juan José Pena Martínez, Jesús Bas Quesada, Felipe Palou Larrañaga, (D) Neeraj K. Yadav, (D) Jasvipul S. Chawla, and John O. Dabiri

\section{COLLECTIONS}

Paper published as part of the special topic on Advances in Wind Plant Controls: Strategies, Implementation, and Validation AWPC2020

F This paper was selected as Featured

SCI This paper was selected as Scilight
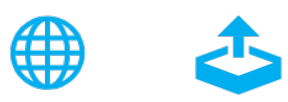

\section{ARTICLES YOU MAY BE INTERESTED IN}

Atmospheric conditions and yaw misalignment significantly impact wind turbine power production

Scilight 2020, 521101 (2020); https://doi.org/10.1063/10.0002969

Data-driven modeling of the wake behind a wind turbine array

Journal of Renewable and Sustainable Energy 12, 033304 (2020); https://

doi.org/10.1063/5.0004393

Comparison of modular analytical wake models to the Lillgrund wind plant

Journal of Renewable and Sustainable Energy 12, 053311 (2020); https://

doi.org/10.1063/5.0018695

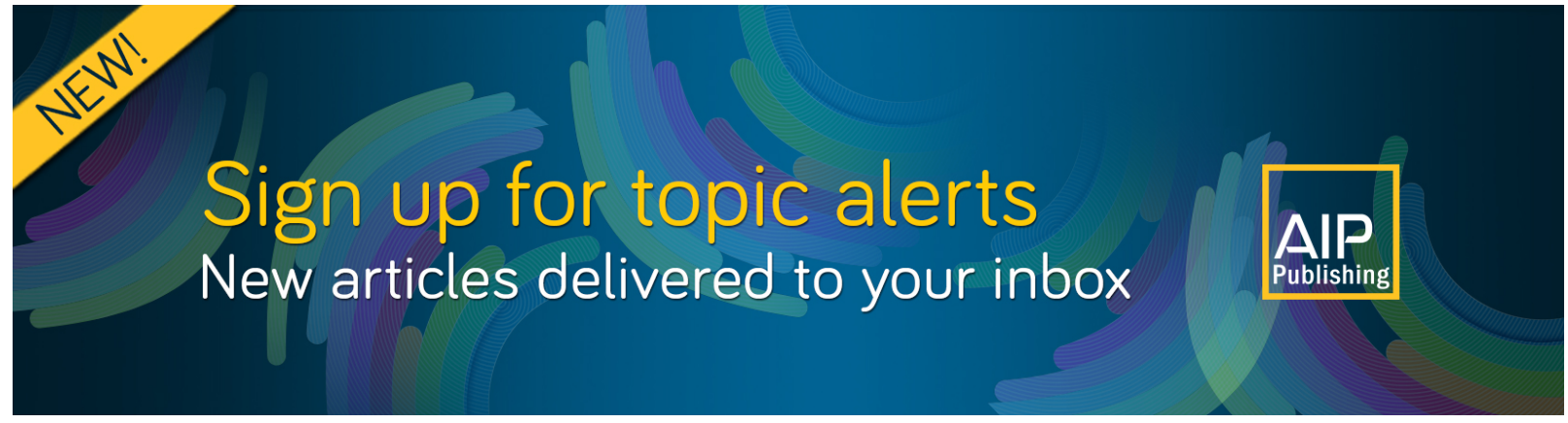




\title{
Influence of atmospheric conditions on the power production of utility-scale wind turbines in yaw misalignment $\mathrm{F}$ (c)
}

Cite as: J. Renewable Sustainable Energy 12, 063307 (2020); doi: 10.1063/5.0023746

Submitted: 1 August 2020 - Accepted: 19 November 2020 •

Published Online: 21 December 2020

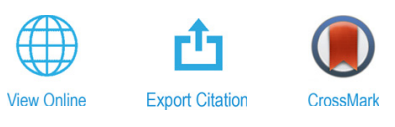

Michael F. Howland, ${ }^{1,2, a)}$ (D) Carlos Moral González, ${ }^{3}$ Juan José Pena Martínez, Jesús Bas Quesada, ${ }^{3}$ Felipe Palou Larrañaga, ${ }^{4}$ Neeraj K. Yadav, ${ }^{5}$ (iD) Jasvipul S. Chawla, ${ }^{5}$ (D) and John O. Dabiri ${ }^{2,6}$

\begin{abstract}
AFFILIATIONS
${ }^{7}$ Civil and Environmental Engineering, Massachusetts Institute of Technology, Cambridge, Massachusetts 02139, USA

${ }^{2}$ Graduate Aerospace Laboratories (CALCIT), California Institute of Technology, Pasadena, California 91125, USA

${ }^{3}$ Siemens Gamesa Renewable Energy Innovation \& Technology, SL. Calle Ramírez de Arellano, 37, 28043 Madrid, Spain

${ }^{4}$ Siemens Gamesa Renewable Energy Innovation \& Technology, SL. Avda. Ciudad de la Innovación, 2, 31621 Sarriguren, Navarra, Spain

${ }^{5}$ ReNew Power Private Limited, Gurugram 122009, Haryana, India

${ }^{6}$ Department of Mechanical and Civil Engineering, California Institute of Technology, Pasadena, California 91125, USA
\end{abstract}

Note: This paper is part of the special issue on Advances in Wind Plant Controls: Strategies, Implementation, and Validation.

a) Author to whom correspondence should be addressed: mhowland@mit.edu

\begin{abstract}
The intentional yaw misalignment of leading, upwind turbines in a wind farm, termed wake steering, has demonstrated potential as a collective control approach for wind farm power maximization. The optimal control strategy and the resulting effect of wake steering on wind farm power production are in part dictated by the power degradation of the upwind yaw misaligned wind turbines. In the atmospheric boundary layer, the wind speed and direction may vary significantly over the wind turbine rotor area, depending on atmospheric conditions and stability, resulting in freestream turbine power production which is asymmetric as a function of the direction of yaw misalignment and which varies during the diurnal cycle. In this study, we propose a model for the power production of a wind turbine in yaw misalignment based on aerodynamic blade elements, which incorporates the effects of wind speed and direction changes over the turbine rotor area in yaw misalignment. The proposed model can be used for the modeling of the angular velocity, aerodynamic torque, and power production of an arbitrary yaw misaligned wind turbine based on the incident velocity profile, wind turbine aerodynamic properties, and turbine control system. A field experiment is performed using multiple utility-scale wind turbines to characterize the power production of yawed freestream operating turbines depending on the wind conditions, and the model is validated using the experimental data. The resulting power production of a yaw misaligned variable speed wind turbine depends on a nonlinear interaction between the yaw misalignment, the atmospheric conditions, and the wind turbine control system.
\end{abstract}

Published under license by AIP Publishing. https://doi.org/10.1063/5.0023746

\section{INTRODUCTION}

Recent work has focused on the development of methodologies to increase the power production of wind farms through collective operation that considers aerodynamic interactions among individual turbines (see, e.g., the study by Kheirabadi and Nagamune ${ }^{1}$ for a recent review). One wind farm control methodology that demonstrates potential in simulations, ${ }^{2,3}$ lab experiments, ${ }^{4,5}$ and field experiments ${ }^{6-9}$ to increase collective turbine power production is wake steering, which entails the intentional yaw misalignment of turbines to deflect wake regions laterally away from downwind generators. The potential of wake steering to increase wind farm power production depends on the magnitude of wake interactions between the wind turbines, the magnitude of the wake deflection as a function of yaw misalignment, and the power production lost by the yaw misaligned turbines. ${ }^{10}$ The power production of a wind turbine in yaw misalignment is often modeled $^{3,11}$ as 


$$
P_{r}=\frac{P_{\gamma}}{P_{\gamma_{0}}} \approx \cos ^{P_{p}}(\gamma)
$$

where $P_{r}$ is the power ratio between the yaw misaligned $P_{\gamma}$ and yaw aligned $P_{\gamma_{0}}$ turbines. The yaw misalignment measured at the wind turbine hub height is given by $\gamma$. The power ratio can also be stated in terms of the coefficient of power $C_{p}=P /\left(\frac{1}{2} \rho A u_{\infty}^{3}\right)$, such that $P_{r}=C_{p}(\gamma) / C_{p}(\gamma=0)$, where $\rho, A$, and $u_{\infty}$ are the fluid density, turbine area, and incident velocity, respectively. Experimental wind tunnel measurements have shown that $P_{p}$ can vary significantly depending on the turbine model and experimental setup. Madsen et $a .^{12}$ and Medici ${ }^{13}$ found that $P_{p} \approx 2$ for experimental turbine models, whereas Dahlberg and Montgomerie ${ }^{14}$ found $1.88<P_{p}<5.14$ at an offshore demonstration facility. Large eddy simulations (LESs) of actuator line model wind turbines ${ }^{15}$ have shown $P_{p} \approx 1.88$ for the NREL $5 \mathrm{MW}$ reference turbine. ${ }^{16}$ Krogstad and Adaramola ${ }^{17}$ found that $P_{p} \approx 3$ for a rotating wind turbine model in wind tunnel experiments with turbulent inflow generated by a static grid. Bartl et al. ${ }^{5,18}$ found that $P_{p} \approx 3$ for a rotating wind turbine model in wind tunnel experiments with low and high turbulence uniform inflow and sheared inflow conditions. Schreiber et al. ${ }^{19}$ and Draper et al. ${ }^{20}$ used wind tunnel experiments and LES to show that $P_{p} \approx 1.8$ for a wind turbine in sheared freestream conditions. Fleming et al. ${ }^{6}$ found $P_{p} \approx 1.4$ for the Envision $4 \mathrm{MW}$ turbine using LES and confirmed this value in a field experiment although the number of data points beyond $|\gamma|>10^{\circ}$ was limited.

Wind turbine modeling methods based on blade element momentum $(\mathrm{BEM})^{12,21}$ or actuator disk theory ${ }^{22}$ both predict that $P_{p}=3$ (see, e.g., recent discussion by Liew et al. ${ }^{11}$ ). Often, BEM methods leverage empirical corrections to improve the agreement with experimental data in yawed conditions (see, e.g., the study by Madsen et $a .^{23}$ ), but these corrections are not necessarily known a priori or generally applicable. The challenge for BEM methods to predict $P_{p}$, or more generally $P_{r}(\gamma)$ or $C_{p}(\gamma)$, necessitates its estimation through computationally expensive LES of wind turbine models (see discussion by Fleming et al. ${ }^{6}$ ).

Engineering wake models are often used for the selection of the optimal yaw misalignment angles for a particular wake steering scenario. ${ }^{24}$ Within the wake models, $P_{p}$ is explicitly parameterized by the user $^{3,6,7}$ or the coefficient of power $C_{p}$ as a function of yaw misalignment must be known a priori, which is a major barrier to wake steering deployment. Accurate estimates of $P_{p}$ are required for the application of wake models for wind farm optimization since $P_{p}$ will dictate the trade-off between the power loss at the upwind turbine against the power gain for the downwind generator. LES studies have shown that an incorrect estimate for $P_{p}$ can lead to suboptimal wake steering performance. ${ }^{25}$ Draper et al. ${ }^{20}$ found that $P_{p}$ for a waked turbine depends on the yaw misalignment of the upwind turbine and fit experimental coefficient of power $C_{p}$ curves to find that $1.3<P_{p}<2.5$. Liew et al. ${ }^{11}$ recently demonstrated by LES that $P_{p}=3$ is a poor estimate for wind turbines in yaw misalignment with complex, non-uniform incident wake flow and found that the value of $P_{p}$ depends on the incident wind conditions. In the atmospheric boundary layer $(\mathrm{ABL})$, the wind speed and direction vary as a function of height due to Coriolis, surface drag, pressure gradient, and other competing forces. ${ }^{26}$ Since the value of $P_{p}$ will depend on the incident wind conditions, $P_{p}$ is not only specific to the turbine make or model but it also has a functional dependence on the wind farm site and time of day, even in freestream operation. This also presents a challenge in the comparison of literature reported values of $P_{p}$ with different turbine models and inflow conditions.

There have been a number of recent wake steering power maximization studies that have noted an asymmetry in the power production of a downwind turbine with respect to the direction of the yaw misalignment of the upwind turbine given full alignment. ${ }^{2,5,27}$ Recent studies have sought to explain the noted asymmetries based on the analysis of wake dynamics. Archer and Vasel-Be-Hagh ${ }^{28}$ hypothesized that this asymmetry was a result of Coriolis forces that cause clockwise wake turning in the northern hemisphere. ${ }^{29,30}$ Gebraad et al. ${ }^{3}$ proposed that this was the result of the clockwise wind turbine blade rotation causing the wake to rotate counterclockwise and introducing a natural rightward deflection with $\gamma=0^{\circ}$ and sheared boundary layer flow. Further, the three-dimensional curled wake effect of yaw misaligned wind turbines $^{31,32}$ may play a role in this asymmetry ${ }^{33-35}$ as well as the influence of wind direction changes over the turbine area on wind turbine wakes. ${ }^{36}$ However, previous studies have not considered a fundamental flow physics mechanism that would result in asymmetric thrust, angular velocity, torque, and power production for a yawed wind turbine operating in freestream conditions depending on the direction of misalignment. As further motivation, in a recent wake steering field experiment, Doekemeijer et al. ${ }^{9}$ found an unexpected asymmetry in $P_{r}$ of a freestream yaw misaligned wind turbine as a function of the sign of $\gamma$.

Aside from collective wake steering control, wind turbines attempting to minimize yaw misalignment through standard operation exhibit natural yaw offsets due to controller errors, ${ }^{37}$ rapidly evolving wind conditions, and a trade-off between yaw error and yaw control actuation. ${ }^{38}$ Understanding and modeling the joint influence of yaw misalignment and the incident wind conditions on wind turbine power production are, therefore, useful for reducing wind farm energy production estimate error and uncertainty. ${ }^{39}$

The primary goal of this article is to develop a simple quantitative model that describes the power ratio $P_{r}(\gamma)$ depending on wind speed and direction variations as a function of height, which evolve during the diurnal cycle at a wind farm. This model will be useful for the prediction of the power production of an arbitrary wind turbine in yaw misalignment depending on the site-specific incident wind conditions and will be directed toward control-oriented wake modeling such as the FLORIS model ${ }^{40}$ or lifting line model. ${ }^{7,41}$ A secondary goal of this article is to perform a detailed, full-scale field experiment to characterize the power ratio $P_{r}$ of a wind turbine in yaw misalignment considering the broad range of realized field wind conditions for the purpose of performing a subsequent full-scale field experiment of wake steering to increase energy production. This field experiment will also serve to validate the presented model for $P_{r}(\gamma)$. This article is organized as follows: in Sec. II, the theoretical influence of the conditions occurring in stable, unstable, and approximately neutral stability states is discussed and a power ratio model is proposed. In Sec. III, the full-scale field experiment design is introduced and the experimental results and model comparisons are made in Sec. IV. The implications of the results on wake steering control, and wind turbine operation more broadly, are discussed in Sec. V, and conclusions are given in Sec. VI.

\section{POWER PRODUCTION MODEL WITH SHEAR AND VEER}

In this section, a model for the joint influence of shear, veer, and yaw misalignment on the power production of a wind turbine is 
proposed. The theoretical influence of atmospheric stability on the shear and veer present in the ABL is discussed in Sec. II A, and the power production model for arbitrary wind profiles is presented in Sec. II B. The influence of the turbine control system is discussed in Sec. II B 1. The model is concisely summarized in Sec. II C. Model results for canonical ABL wind profiles are given in Sec. II D.

\section{A. Theoretical consideration of stratification on the ABL shear and veer}

The magnitude of the wind direction change as a function of height depends on the atmospheric conditions. The wind direction change over the wind turbine rotor area is defined as

$$
\Delta \alpha=\alpha\left(z=z_{h}+R\right)-\alpha\left(z=z_{h}-R\right),
$$

where $R$ is the wind turbine radius and $\alpha(z)$ is the wind direction with $0^{\circ}$ corresponding to north and proceeding clockwise. The wind turbine hub height is $z_{h}$. Veer conditions result in $\Delta \alpha>0^{\circ}$ (e.g., flow below the hub height directed to the northeast and flow above the hub height directed to the east), and backing is defined as $\Delta \alpha<0^{\circ}$. The wind direction change is taken as the shortest rotational path from $z_{h}-R$ to $z_{h}+R$. The wall normal coordinate is $z$, and $x$ and $y$ are the horizontal directions. The wind speeds in the $x, y$, and $z$ directions are $u, v$, and $w$, respectively. The robust characterization of $\Delta \alpha$ [Eq. (2)] relies on monotonic behavior in the wind direction $\alpha(z)$ over the wind turbine rotor area as is commonly the case in flat terrain atmospheric boundary layer flows driven by a pressure gradient and Coriolis forces. ${ }^{42}$ However, the model derived in Sec. II will not enforce this approximation, which is useful from a theoretical perspective, and therefore, arbitrary wind direction profiles can be used. The wind direction over the wind turbine rotor area will be characterized in the experimental field data in Sec. III A.

The wind direction change $\Delta \alpha$ depends on the effects of stratification, which is the measure of the ambient density changes in the atmosphere due to temperature and pressure variations. ${ }^{42}$ With unstable stratification, convective ABL conditions present and the wind direction change as a function of height will be $\Delta \alpha \approx 0^{\circ}$ due to enhanced vertical mixing, which reduces velocity gradients. ${ }^{26}$ In the limit of neutral stratification (constant density in the atmosphere) with a balance of a geostrophic pressure gradient, Coriolis forces, and surface stress, and invoking an eddy viscosity model, the flow becomes the Ekman layer, which is governed by

$$
\begin{gathered}
-f_{c} v=\frac{-1}{\rho} \frac{\partial P}{\partial x}+\nu_{t} \frac{\partial^{2} u}{\partial z^{2}}, \\
f_{c} u=\frac{-1}{\rho} \frac{\partial P}{\partial y}+\nu_{t} \frac{\partial^{2} v}{\partial z^{2}},
\end{gathered}
$$

and a hydrostatic balance in the vertical direction, where $f_{c}=2 \omega$ $\sin (\phi)$ is the Coriolis parameter, $\phi$ is the latitude, $\omega$ is the angular velocity of Earth, $P$ is the pressure, $\rho$ is the density of the air, and $\nu_{t}$ is the eddy viscosity. With a fixed eddy viscosity as a function of $z$, the solution is given analytically ${ }^{42}$ as

$$
\begin{gathered}
u=G\left(1-e^{-z / \delta} \cos (z / \delta)\right), \\
v=G e^{-z / \delta} \sin (z / \delta),
\end{gathered}
$$

where $G$ is the geostrophic wind speed magnitude and $\delta=\sqrt{2 \nu_{t} / f_{c}}$ is the Ekman layer depth. The geostrophic wind is the wind associated with an exact balance between the geostrophic pressure gradient and Coriolis forces in the free atmosphere. The Ekman layer wind direction is given by

$$
\alpha_{E}(z)=\tan ^{-1}\left(\frac{v(z)}{u(z)}\right)=\tan ^{-1}\left(\frac{e^{-z / \delta} \sin (z / \delta)}{1-e^{-z / \delta} \cos (z / \delta)}\right) .
$$

The wind direction variation as a function of height in this flow is termed the Ekman spiral; the wind vector turns to the left, or counterclockwise, moving toward $z=0$, resulting in veer conditions of $\Delta \alpha>0$. The eddy viscosity can be qualitatively modeled using a mixing length model, $l_{m}=\kappa z /(1+\kappa z / \lambda)$ and $\nu_{t}=\kappa l_{m} u_{\tau}$, with $\lambda=\mathcal{O}(10)$ meters, the maximum value of $l_{m}$ in the free atmosphere, ${ }^{43}$ von Kármán constant $\kappa$, and a friction velocity $\boldsymbol{u}_{\tau} \approx 0.5 \mathrm{~m} / \mathrm{s}$, giving $\nu_{t} \approx 2 \mathrm{~m}^{2} / \mathrm{s}$, a reasonable value for mid-latitudes. ${ }^{44}$ Overall, at a latitude of $\phi \approx 25^{\circ} \mathrm{N}$, the approximate latitude of interest for the experimental wind farm, this returns a veer between the rotor diameter extent of $\Delta \alpha \approx 6^{\circ}$ for a wind turbine with a diameter of approximately $100 \mathrm{~m}$. Further, as a result of Coriolis forces, the maximum speed in the Ekman layer occurs at a finite value of $z$ and is larger in magnitude than the geostrophic wind speed; this is termed the subgeostrophic or low-level jet, which is also present in stable ABL conditions as a result of the suppression of turbulent stresses and inertial oscillations. ${ }^{45,46}$ Wind conditions in the atmosphere differ from the Ekman layer solution due to stratification and since the ABL is not statistically stationary (several multiples of $1 / f_{c}$ are required for the Ekman layer flow to reach a statistically stationary state ${ }^{42,47}$ during which the ABL state typically transitions). Wind speed and direction variations as a function of height significantly modify wind farm power production through an influence on the wake recovery ${ }^{48}$ and individual turbine performance. 49,50

In stable stratification, the veering effect increases due to the suppression of turbulent production and a reduction in the boundary layer height. ${ }^{51,52}$ Deusebio et al. ${ }^{53}$ used direct numerical simulations of stable Ekman layers and found that the veering angle generally increases with $u_{*} / L f_{c}$, where

$$
L=-\frac{u_{*}^{3} \overline{\theta_{T}}}{\kappa g \overline{w^{\prime} \theta_{T s}^{\prime}}}
$$

is the Obhukhov length with potential temperature $\theta_{T}$, friction velocity $u_{*}$, and gravity $g$. The surface heat flux is denoted by $\overline{w^{\prime} \theta_{T s}^{\prime}}$. As the strength of the stability increases, $L$ is positive and decreases in magnitude, and the veering angle will generally increase. In summary, during unstable conditions that typically occur during the day, the veer will be approximately zero, while during stable conditions that occur at night, veer and a sub-geostrophic jet will present. With increasing stable stratification, the veering angle is expected to increase. Characteristic power law and Ekman layer velocity profiles are shown in a wind turbine side view in Fig. 1(a). The wind direction shear associated with the Ekman layer (veer) is shown qualitatively in a wind turbine front view in Fig. 1(b).

\section{B. Blade element power ratio model}

In yaw aligned operation with spatially uniform inflow, as a wind turbine blade rotates around its central axis of rotation, the blade angle of attack does not depend on the azimuthal position. In yaw 
Side view

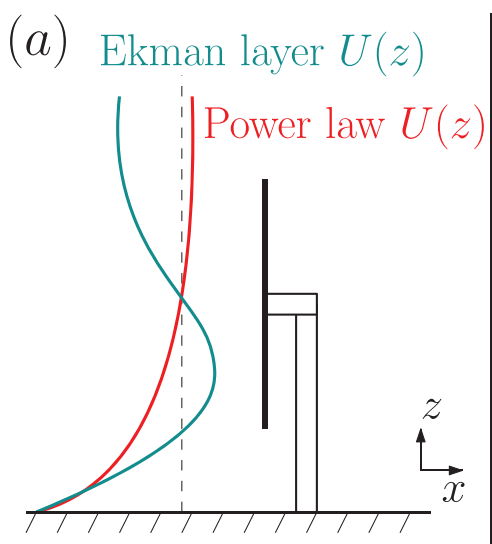

Front view

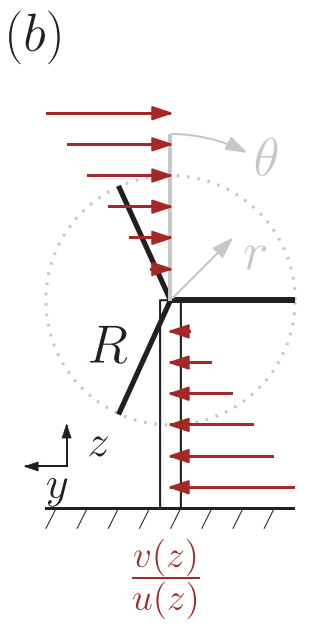

Top view

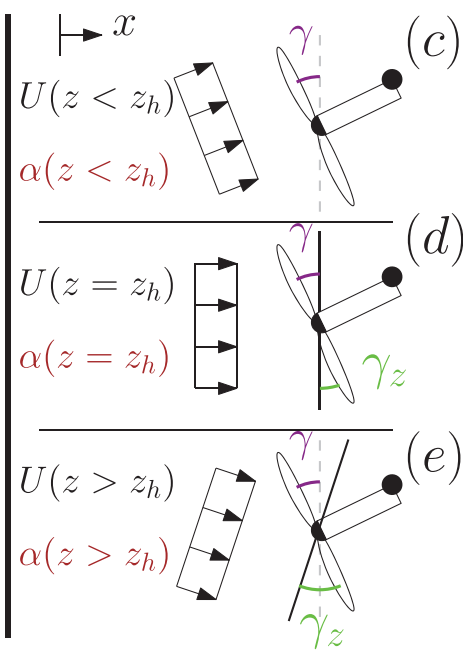

FIG. 1. (a) Side view of a yaw aligned wind turbine. The incident wind is sheared and skewed with the wind speed $U(z)$ and direction $\alpha(z)$ depending on the height in the atmospheric boundary layer. Two example wind speed profiles indicative of the canonical power law and Ekman layer behavior are shown. The Ekman layer manifests as a balance between Coriolis, pressure gradient, and surface drag forces. (b) Front view of a yaw aligned wind turbine with a positively veered incident inflow wind indicated by the normalized incident spanwise velocity $v(z) / u(z)$. The spanwise velocity profile is shown for illustrative purposes and is not generally linear. (c)-(e) Top view of a positively yaw misaligned wind turbine, which is a counterclockwise rotation viewed from above. The wind turbine hub height is indicated by $z_{h}$. The top view slice is taken (c) below the hub height $\left(z<z_{h}\right)$, (d) at the hub height $\left(z=z_{h}\right)$, and $(e)$ above the hub height $\left(z>z_{h}\right)$. The yaw misalignment is characterized by $\gamma=\alpha\left(z=z_{h}\right)-\beta$, the angle between the nacelle position $\beta$ and the hub height wind direction, $\alpha\left(z=z_{h}\right)$. The local yaw misalignment angle at the particular location of $z$ is given by $\gamma_{z}=\alpha(z)-\beta$. Given positive hub height yaw misalignment and positive veer conditions associated with Coriolis effects in the Northern Hemisphere, the wind turbine is locally more aligned below the hub height $\left(z<z_{h}\right)$ and less aligned above the hub height $\left(z>z_{h}\right)$. The black circle on the wind turbine nacelle is the wind speed anemometer.

misalignment, sheared conditions, veered conditions, or any combination of the three, the angle of attack has a functional dependence on the azimuthal position. Following blade element theory, we can derive a quantitative model that captures this consequence. Kragh and Hansen ${ }^{54}$ developed a model for the influence of shear on the axial forces acting on a wind turbine in yaw misalignment. Here, we use the derivation of Kragh and Hansen ${ }^{54}$ as a starting point and generalize the analysis to veered conditions and for power production estimation. This analytical model is used as a starting point rather than a more complex aeroelastic solver in order to establish the first-order effects of the incident wind conditions and yaw misalignment in a complex engineering system.

Yaw alignment controllers leverage measurements of the wind direction by nacelle-mounted wind vanes in order to correct offsets between the wind direction and the nacelle position. ${ }^{37}$ This characterization of yaw is, therefore, defined as the difference between the nacelle position and the wind direction measured at the hub height by the wind vane,

$$
\gamma=\alpha\left(z=z_{h}\right)-\beta,
$$

where $\gamma$ is the yaw misalignment, $\beta$ is the nacelle position, and $\alpha\left(z=z_{h}\right)$ is the wind direction at hub height $z=z_{h}$. As discussed in Sec. II A, $\alpha(z)$ may have a functional dependence on $z$, the height above the ground, in the atmospheric boundary layer.

The wind speed incident on a blade segment is a function of its angular velocity and the incident wind velocity vector. Neglecting the tangential induction factor (see, e.g., the study by Kragh and Hansen ${ }^{54}$ ), the tangential velocity incident to the blade is

$$
u_{\tau}(r)=\Omega r
$$

where $\Omega$ is the angular velocity of the blade and $r$ and $\tau$ are the radial and tangential directions, respectively. The azimuthal angle is $\theta$, and $x$ is the axial direction. Side, front, and top views of a wind turbine with the coordinate geometry used in this study are shown in Fig. 1. The blade sectional view and corresponding coordinate system for the blade element model are shown in Fig. 2.

The local inflow direction over the rotor area is modified by the yaw misalignment angle in addition to wind direction variations as a function of $z$. A local misalignment angle is defined as

$$
\gamma_{z}(r, \theta)=\alpha(r, \theta)-\beta
$$

which is a function of the wind direction that varies over the rotor area $\alpha(r, \theta)$ and the wind turbine nacelle position $\beta$. The wind speed vector is, assuming negligible tilt, ${ }^{54}$

$$
\vec{v}_{\text {wind }}=\left[\begin{array}{c}
\cos \left(\gamma_{z} \cos (\theta)\right) \hat{x} \\
-\sin \left(\gamma_{z} \cos (\theta)\right) \hat{\tau}
\end{array}\right] U \cos \left(\gamma_{z} \sin (\theta)\right),
$$

where $U(r, \theta)$ is the inflow wind speed corrected for axial induction effects. The azimuthal variation of the axial induction factor is neglected in this study but could be incorporated (e.g., using Glauert's empirical correction ${ }^{55}$ ) in future work without loss of generality. Note 


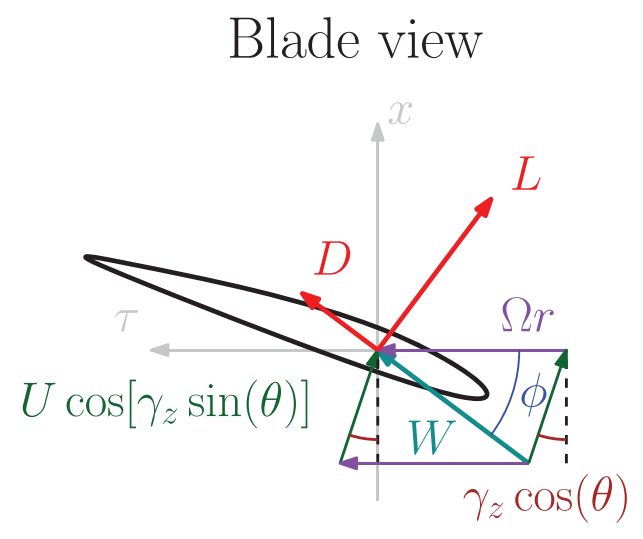

FIG. 2. Blade sectional view of a positively yaw misaligned wind turbine, which is a counterclockwise rotation viewed from above (see Fig. 1). The axial and tangential directions are $x$ and $\tau$, respectively. The lift and drag forces are indicated by $L$ and $D$, respectively. The turbine yaw misalignment is characterized by $\gamma=\alpha\left(z=z_{h}\right)$ $-\beta$, the misalignment angle between the nacelle position $\beta$ and the hub height wind direction, $\alpha\left(z=z_{h}\right)$, where $z_{h}$ is the hub height. The local yaw misalignment angle incident to the blade section at the particular location of $(r, \theta)$ is given by $\gamma_{z}(r, \theta)=\alpha(r, \theta)-\beta$. The blade view shows a cross section of a wind turbine blade as it passes through $\theta=0^{\circ}$.

that the inflow wind speed $U(z)$ is transformed into polar coordinates $(r, \theta)$ corresponding to the rotor plane.

The squared relative wind speed is

$$
\begin{aligned}
W^{2}(r, \theta)= & {\left[U \cos \left(\gamma_{z} \sin (\theta)\right) \cos \left(\gamma_{z} \cos (\theta)\right)\right]^{2} } \\
& +\left[\Omega r-U \cos \left(\gamma_{z} \sin (\theta)\right) \sin \left(\gamma_{z} \cos (\theta)\right)\right]^{2},
\end{aligned}
$$

and the inflow angle $\phi$ is

$$
\phi=\tan ^{-1}\left(\frac{U \cos \left(\gamma_{z} \sin (\theta)\right) \cos \left(\gamma_{z} \cos (\theta)\right)}{\Omega r-U \cos \left(\gamma_{z} \sin (\theta)\right) \sin \left(\gamma_{z} \cos (\theta)\right)}\right) .
$$

The axial force at a particular radial section is ${ }^{54}$

$$
d f_{x}=\frac{1}{2} \rho c W^{2}\left[C_{L}(\phi-\psi) \cos (\phi)+C_{D}(\phi-\psi) \sin (\phi)\right] d r
$$

where $\psi$ incorporates the blade pitch and twist at the local radial section, $c$ is the chord length, $\rho$ is the density of the incident air, and $C_{L}$ and $C_{D}$ are the lift and drag coefficients evaluated at an angle of attack of $\phi-\psi$. The tangential force at a particular radial section is ${ }^{56}$

$$
d f_{\tau}=\frac{1}{2} \rho c W^{2}\left[C_{L}(\phi-\psi) \sin (\phi)-C_{D}(\phi-\psi) \cos (\phi)\right] d r .
$$

The incremental torque at the particular radial section is given by

$$
d T=r d f_{\tau},
$$

and therefore, the incremental contribution to the wind turbine power production is

$$
d P=\Omega d T,
$$

where the angular velocity of the blades $\Omega$ is not a function of $r$ or $\theta$. Equation (18) is used to compute the power ratio, defined as the power production of a yaw misaligned turbine with respect to a yaw aligned turbine,

$$
P_{r}=\frac{P_{\gamma}}{P_{\gamma_{0}}}=\frac{\Omega_{\gamma} \int_{0}^{2 \pi} \int_{0}^{R} c r W_{\gamma}^{2}\left[C_{L}\left(\phi_{\gamma}-\psi\right) \sin \left(\phi_{\gamma}\right)-C_{D}\left(\phi_{\gamma}-\psi\right) \cos \left(\phi_{\gamma}\right)\right] d r d \theta}{\Omega_{\gamma_{0}} \int_{0}^{2 \pi} \int_{0}^{R} c r W_{\gamma_{0}}^{2}\left[C_{L}\left(\phi_{\gamma_{0}}-\psi\right) \sin \left(\phi_{\gamma_{0}}\right)-C_{D}\left(\phi_{\gamma_{0}}-\psi\right) \cos \left(\phi_{\gamma_{0}}\right)\right] d r d \theta}
$$

where the subscripts of $\gamma$ and $\gamma_{0}$ denote a wind turbine that is yaw misaligned and yaw aligned with respect to the hub height wind direction, respectively. The angular velocity ratio is defined as

$$
\Omega_{r}=\frac{\Omega_{\gamma}}{\Omega_{\gamma_{0}}}
$$

and correspondingly, the torque ratio is given as

$$
T_{r}=\frac{T_{\gamma}}{T_{\gamma_{0}}}=\frac{P_{r}}{\Omega_{r}} .
$$

In order to model the power ratio, incident wind speed $U(z)$ and direction $\alpha(z)$ profiles are required, in addition to the turbine-airfoilspecific coefficients of lift and drag and blade twist and pitch. In general, for best quantitative accuracy, the lift, drag, twist, and chord tables for the specific wind turbine of study should be used if available. For simplicity, the lift and drag coefficients, corrected for threedimensional effects, for the NACA64 airfoil reported for the NREL
$5 \mathrm{MW}$ reference turbine are used ${ }^{16}$ in Sec. II D. The aerodynamic properties of the experimental turbine of interest are used in Sec. IV for the field data comparisons. Compared to aeroelastic solvers, the simple, computationally efficient model given by Eq. (19) captures the leading order effects of yaw misalignment and the incident wind conditions and can be leveraged for rapid prototyping or control-oriented optimization to predict $P_{r}(\gamma)$. The proposed model does not include the assumptions associated with the momentum component of BEM theory, which require empirical skewed wake corrections (see discussion by Moriarty and Hansen ${ }^{57}$ ). The model will be applied to canonical ABL wind profiles in Sec. II D and experimentally measured wind speed $U(z)$ and direction $\alpha(z)$ profiles in Sec. IV.

\section{Wind turbine generator torque control}

Given velocity and wind direction profiles, the power ratio can be predicted using Eq. (19) and $\Omega_{r}$. The angular 
velocity of the blades is normalized and given by the tipspeed ratio (TSR),

$$
\lambda=\frac{\Omega R}{u_{\infty}} .
$$

In yaw aligned operating conditions, an optimal tip-speed ratio exists such that $C_{p}$ is maximized for given inflow conditions. The angular velocity, and therefore tip-speed ratio, of a wind turbine in yaw misalignment depends on the control system in use. Bastankhah and Porté-Agel $^{58}$ found that the power ratio, and $P_{p}$ factor, of a wind turbine in yaw misalignment is dependent on the tip-speed ratio. Medici $^{13}$ used a model wind turbine embedded in a wind tunnel and found that the tip-speed ratio $\lambda_{\gamma} / \lambda_{\gamma_{0}} \sim \cos (\gamma)$ and the power ratio $P_{r} \sim \cos ^{2}(\gamma)$, implying that $T_{r} \sim \cos (\gamma)$. Bartl et al. ${ }^{5,18}$ fixed the tip-speed ratio between yaw aligned and misaligned cases and found that $P_{r} \sim \cos ^{3}(\gamma)$ in wind tunnel experiments. Bastankhah and Porté-Agel ${ }^{58}$ found $P_{r} \sim \cos ^{3}(\gamma)$ for a wind turbine operating at its optimal tip-speed ratio, implying that the optimal tip-speed ratio was fixed for the various yaw misalignment angles and $\Omega_{r} \sim \cos (\gamma)$. In a following study, Bastankhah and Porté-Agel ${ }^{59}$ tabulated the optimal $\Omega_{\gamma}$ (in rotations per minute) that returned the maximum power production as a function of the incident wind conditions and the yaw misalignment of the model turbine in wind tunnel sheared inflow. The optimal $\Omega_{\gamma}$ appeared to have a weak dependence on the yaw misalignment angle (Fig. 2 in the study by Bastankhah and PortéAgel $\left.^{59}\right)$. Based on the model turbine's local wind condition measurements, the optimal setpoint of $\Omega$, was found from the lookup table and applied to the yaw misaligned turbine and $P_{p} \approx 2.5$ although only positive yaw angles were shown. For these small-scale experimental model wind turbines, the operational angular velocity would be specified to Eq. (19) and $P_{r}(\gamma)$ could be predicted based on the incident wind conditions.

For variable speed utility-scale horizontal axis wind turbines, the generator torque and pitch angle setpoints are specified based on the wind conditions. ${ }^{16}$ The setpoints are designed to achieve a targeted tip-speed ratio although the steady state angular velocity, or tip-speed ratio, is a consequence of the difference between the aerodynamic and generator torque rather than an explicitly set value. Therefore, the angular velocity achieved will depend on the generator torque setting; these two values will, in turn, dictate the power production. Given a specification of $T_{r}$, Eq. (17) can be used to compute the optimal value of $\Omega_{r}$ to minimize the difference between the prescribed $T_{r}$ and the model prediction for $T_{r}$. In general, for wind conditions below rated wind speeds, modern wind turbines use a proportional-integral (PI) controller on the generator torque to operate at the tip-speed ratio setpoint. ${ }^{60}$ In the present study, the wind turbine generator control system is modified in yaw misalignment. We further assume that the torque controller has reached the steady-state, and therefore, $T_{c}$ (the generator torque) is balanced exactly by the aerodynamic torque (accounting for mechanical losses and the gear-box ratio). Equation (21) gives the aerodynamic torque as a function of the blade angular velocity, and $T_{c}=K \Omega^{2}$ gives the generator controller torque ${ }^{16}$ as a function of the angular velocity (approximation of generator torque control). Altogether, they provide a system of two equations and two unknowns $\left[\Omega_{r}\right.$ and $T_{r}\left(\Omega_{r}\right)$ ] and can be solved with a nonlinear optimization routine [e.g., fminsearch() in Matlab ${ }^{61}$ ].

\section{Blade element model summary}

The power-yaw relationship model proposed in Secs. II B and II B 1 is concisely summarized in this section. A schematic of the control-oriented power ratio model is shown in Fig. 3. The model inputs are categorized as wind turbine details, turbine controller details, and wind conditions. Wind turbine details including the rotor diameter $D$ and hub height $z_{h}$ are required. Additionally, turbine specific airfoil properties including the lift and drag coefficient tables $C_{L}$ and $C_{D}$, chord $c$, and blade pitch and twist $\psi$ are required for each radial section along the wind turbine blade. From the wind turbine internal controller, the tip-speed ratio (TSR) setpoints for yaw aligned operation $\lambda_{\gamma_{0}}$ and the generator torque control system are required. Finally, the wind speed $u(z)$ and direction $\alpha(z)$ profiles as a function of the vertical distance from the ground $z$ are required. The wind profiles can be measured by a profiling LiDAR or a meteorological (MET) mast. The proposed model does not depend on any empirical parameters that require calibration, only information of the wind turbine, and the wind profile.

With the relevant inputs provided, the model computes the angular velocity for yaw misaligned operation $\Omega_{\gamma}$ by equating the aerodynamic torque with the generator torque $T_{a}\left(\Omega_{\gamma}\right)=T_{c}\left(\Omega_{\gamma}\right)$. The aerodynamic torque $T_{a}$ is computed using Eq. (17) integrated over the rotor area, and the generator torque control setpoint $T_{c}$ is a function of the control system and angular velocity $\Omega_{\gamma}$. The power ratio $P_{r}$ is computed using the wind turbine angular velocities $\Omega_{\gamma}, \Omega_{\gamma_{0}}$ in Eq. (19).

There are several model assumptions and simplifications used to arrive at the control-oriented $P_{r}$ model in Eq. (19). The model is quasi-steady such that the aerodynamic torque is exactly balanced by the generator torque control and the wind profiles are provided by a finite-time average. Tilt is neglected, and the axial induction is assumed to be uniform over the rotor area. The tangential induction is neglected. ${ }^{54}$ No assumption on the wind profiles is enforced in the derivation, and therefore, arbitrary wind profiles measured in field conditions may be implemented.

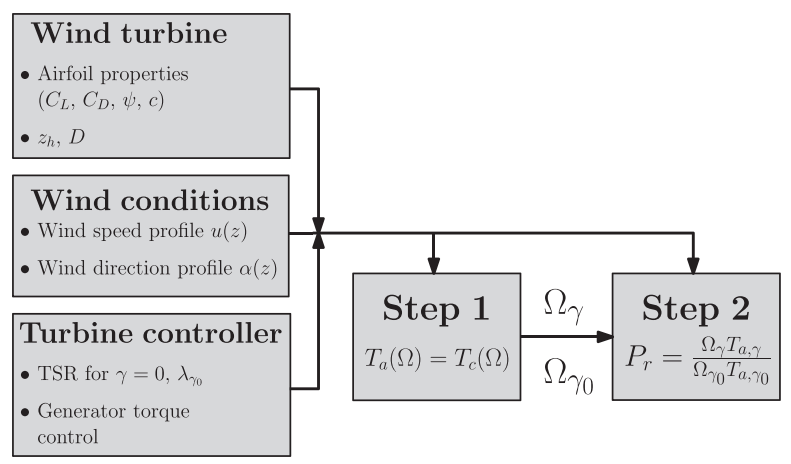

FIG. 3. Schematic of the control-oriented power-yaw relationship power ratio model. The wind turbine, turbine controller, and wind condition details are provided in step 1 that computes the angular velocities for the wind turbine blades for yaw aligned $\Omega_{\gamma_{0}}$ and yaw misaligned $\Omega$, operation. Step 1 equates the generator torque $T_{c}$ to the aerodynamic torque $T_{a}$ to calculate the angular velocity $\Omega$. The turbine and wind condition information and angular velocities are provided in step 2 that uses Eq. (19) to compute the power ratio $P_{r}=P(\gamma) / P\left(\gamma_{0}\right)$. 


\section{Model predictions with canonical ABL wind profiles}

In this section, the model proposed in Sec. II B will be coupled with canonical $\mathrm{ABL}$ wind profiles to establish a qualitative physical expectation before the presentation of field results in Sec. IV. The wind velocity profile is approximated as a power law

$$
u(z)=u_{h}\left(z / z_{h}\right)^{\alpha_{v}},
$$

where $\alpha_{v}$ is the shear exponent and the velocity and vertical location of the wind turbine hub are given by $u_{h}$ and $z_{h}$, respectively. While stratified ABL flows often deviate from power or logarithmic velocity profiles with the development of sub-geostrophic or low-level jets which arise from Coriolis and pressure gradient forces, ${ }^{62}$ the power law is, nevertheless, useful for a first-order approximation. ${ }^{26}$ In order to reveal a qualitative impact of the wind direction shear, in this section, we assume a linear profile in the wind direction as a function of height such that

$$
\frac{d \alpha(z)}{d z}=\frac{\alpha\left(z_{h}+R\right)-\alpha\left(z_{h}-R\right)}{\left(z_{h}+R\right)-\left(z_{h}-R\right)},
$$

where $\alpha\left(z_{h}+R\right)$ and $\alpha\left(z_{h}-R\right)$ are prescribed to give the veer over the turbine face. In the comparison of the proposed model with experimental data in Sec. IV, no assumption on the wind direction profile is made and the experimentally measured velocity profiles are used.

The results from the model given by Eq. (19) for the power law and linear veer profiles and the generator torque control described in
Sec. II B 1 are shown in Figs. 4(a)-4(c). For comparison, the power ratio model results for a prescribed $\Omega_{r}=\cos (\gamma)$ are shown for the same inflow wind profiles in Figs. 4(d)-4(f). Considering the realistic control case with prescribed torque controller $K$, with no shear or veer $\alpha_{v}=\Delta \alpha=0$, the resulting angular velocity ratio $\Omega_{r} \approx 1$ for all yaw misalignment values. However, when shear and veer are incorporated, $\alpha_{v}=0.3, \Delta \alpha=30^{\circ}$, and asymmetry is introduced into $\Omega_{r}$, such that the angular velocity is higher for negative yaw misalignment than for positive yaw. Correspondingly, the torque is also larger for negative yaw misalignment compared to positive yaw, and as a result, the power ratio $P_{r}$ is asymmetric, with $\gamma<0$ producing more power than $\gamma>0$. This result agrees with the qualitative expectation that given positive shear and veer, there is more energy available above the hub height than below the hub height, and negative yaw misalignment will reduce the relative misalignment above the hub location. Further, the power loss due to yaw misalignment cannot be approximated by a simple cosine model [as shown in Eq. (1)], as $P_{r}>\cos ^{2}(\gamma)$ for $\gamma<0$ and $P_{r}<\cos ^{2}(\gamma)$ for $\gamma>0$. On the other hand, when $\alpha_{v}=-0.3$ and $\Delta \alpha=30^{\circ}$, the opposite qualitative asymmetry occurs although the asymmetry is quantitatively different due to the asymmetric effects of the blade rotation direction. While a power law form with $\alpha_{v}=-0.3$ is not likely to occur often in ABL observations, this result serves to approximate the influence of the sub-geostrophic jet, which results in negative shearing conditions.

With $\Omega_{r}=\cos (\gamma)$, an asymmetry is present, but less pronounced. Further, the power ratio approximately follows $\cos ^{3}(\gamma)$ for

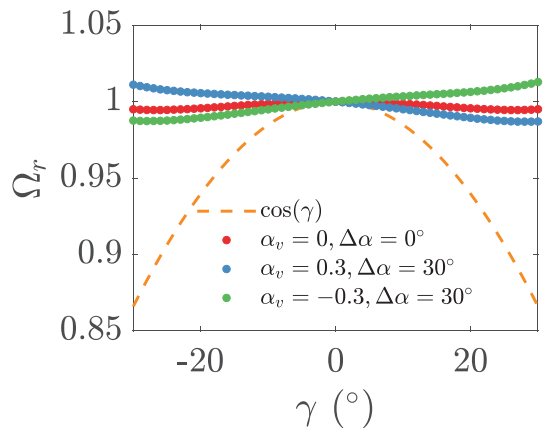

(a)

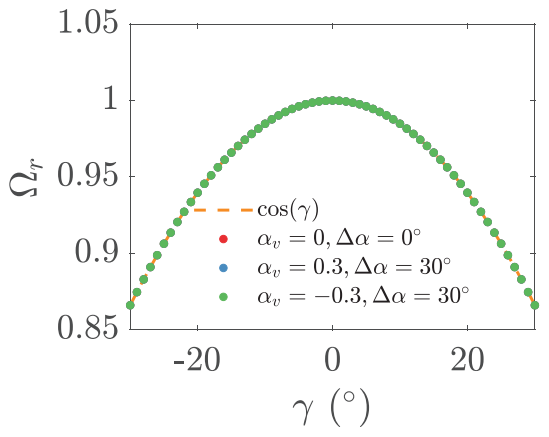

(d)

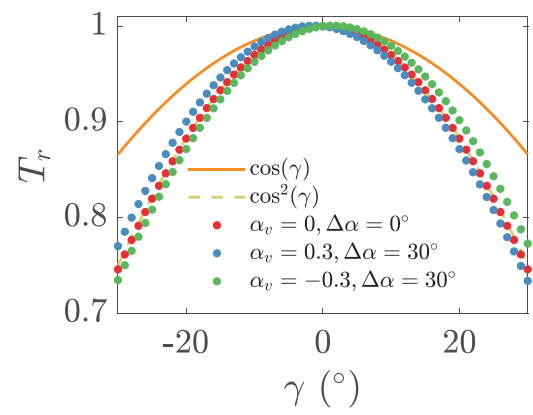

(b)

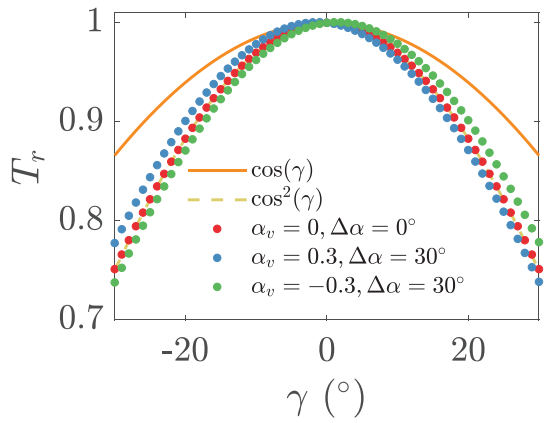

(e)

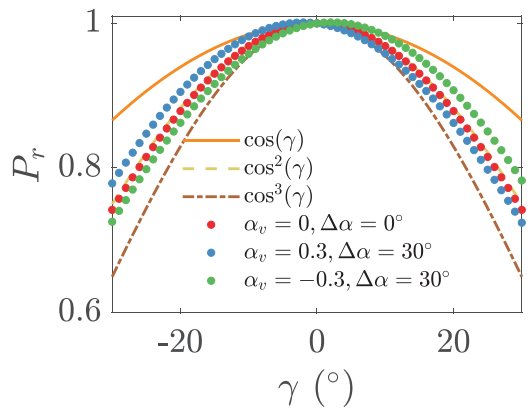

(c)

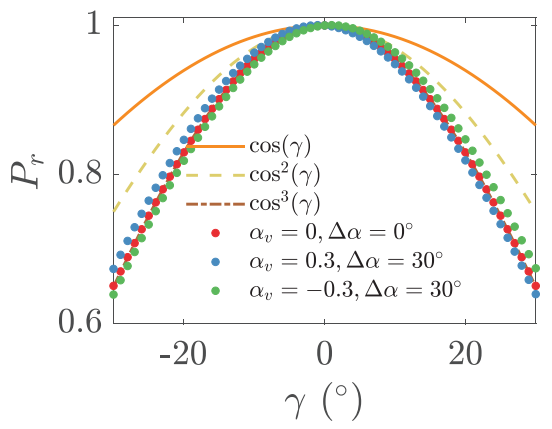

(f)

FIG. 4. Model results with a power-law velocity profile and a linear veer profile. (a) $\Omega_{r}$ (b) $T_{r}$, and (c) $P_{r}$. The angular velocity $\Omega_{\gamma}$ is computed for each yaw misalignment angle by minimizing the difference between the aerodynamic and generator torques. (d)-(f) The same as (a)-(c) except the angular velocity ratio is prescribed as $\Omega_{r}=\Omega_{\gamma} / \Omega_{\gamma_{0}}=\cos (\gamma)$. 
all inflow profile cases. This result confirms the expectation that the power ratio quantity $P_{r}(\gamma)$ will depend on the incident wind conditions and the control system specific to the wind turbine although the model presented in Sec. II B can be used with arbitrary control laws or incident velocity profiles. The model is compared with field experimental data in Sec. IV using the control system for the presently studied wind turbines and the measured incident wind conditions.

\section{WIND FARM AND EXPERIMENTAL SETUP}

The wind farm of interest is located in northwest India. The site contains nearly 100 utility-scale wind turbines of various original equipment manufacturer (OEM) constructions. The wind turbines have diameters and hub heights of approximately $100 \mathrm{~m}$. The wind farm topography is flat with a gradual slope increasing in elevation from northwest to southeast by approximately $100 \mathrm{~m}$ over approximately $25 \mathrm{~km}$. The wind turbine layout for the cluster of interest is shown in Fig. 5(a).

The wind farm is characterized by two distinct wind seasons. The summer corresponds to the Indian monsoon season ${ }^{63}$ for which the wind speeds, as characterized by a site meteorological (MET) mast, are greater than 5 and $10 \mathrm{~m} / \mathrm{s}$ approximately $87 \%$ and $18 \%$ of the time, respectively. The prevalent wind direction during the summer wind season is from the southwest. The other seasons are characterized by incident winds from the west, north, and northeast. By contrast, the non-monsoon wind speeds are greater than 5 and $10 \mathrm{~m} / \mathrm{s}$ approximately $70 \%$ and $13 \%$ of the time, respectively.

Cluster A [see Fig. 5(a)] is in the northern region of the wind farm and is not affected by wind turbine wakes for flows that occur during the non-monsoon wind season. The 2016-2018 yearly averaged monthly wind roses for the non-monsoon season measured by a MET mast approximately $20 \mathrm{~km}$ west of cluster A are shown in Fig. 5(b).

In order to measure the velocity profiles as a function of height incident on cluster A, a Leosphere Windcube V2.0 profiling LiDAR was installed at the field site. The pulsed LiDAR measures backscatter by aerosols in the atmosphere and translates the measurements into a corresponding Doppler shift in order to provide information about the wind speed and direction. The wind speed and direction have measurement uncertainties of $0.1 \mathrm{~m} / \mathrm{s}$ and $2^{\circ}$, respectively. The measurement precisions for the wind speed and direction are $0.005 \mathrm{~m} /$ $s$ and $0.005^{\circ}$. During the non-monsoon wind season, the LiDAR measures the wind speed and direction profiles upwind of turbine $A 1$. The profiling LiDAR measures the velocity at 12 range gates as a function of height between 43 and $200 \mathrm{~m}$ of elevation. A range gate is set at 104 $\mathrm{m}$ to measure the velocity near the hub height.

The experiment was performed from February 12, 2020, until April 7, 2020. In order to characterize the influence of yaw misalignment on freestream wind turbines, six full-scale wind turbines were provided with a yaw misalignment actuating sequence as a function of time [Fig. 6(a)]. Turbine cluster A, the focus of this experiment given the nearby location of the LiDAR, with the actuating and reference turbines highlighted, is shown in Fig. 5(a). For each turbine cluster, threshold wind condition parameters are set for which the yaw actuating time series would be followed. The thresholds were prescribed as wind speeds in Region II of the turbine power curves $\left(C_{p}\right.$ maximizing region) as well as an arc of the incident wind direction such that both the actuating and reference turbines are in freestream wind conditions with no upwind turbines within $20 \mathrm{D}$. If the wind condition threshold values were violated, the actuating wind turbines' supervisory control and data acquisition (SCADA) commands $\gamma_{c}=0$, where $\gamma_{c}$ is the commanded yaw misalignment value.

The realized one-minute averaged yaw misalignment $\gamma_{l}=\alpha_{\mathrm{LiDAR}}$ $\left(z=z_{h}\right)-\beta$ is computed as a difference between the wind turbine nacelle position and the LiDAR wind direction at the hub height. The yaw misalignment is also characterized by the wind turbine, where $\gamma_{t}$ is measured by a nacelle-mounted wind vane and reported as a relative wind direction with respect to the nacelle position orientation. A histogram of the resulting yaw misalignment values for turbine $A 1$, where the yaw misalignment is computed by the turbine $\left(\gamma_{t}\right)$ or by the upwind profiling $\operatorname{LiDAR}\left(\gamma_{l}\right)$ is shown in Fig. 6(b). An example time series from the yaw misalignment field experiment is shown in Fig. 6(c). The SCADA applied yaw misalignment $\gamma_{a}$ attempts to follow Fig. 6(a), provided that the threshold conditions are met. The LiDAR and wind turbine characterized ten-minute moving averaged yaw misalignment values, $\tilde{\gamma}_{l}$ and $\tilde{\gamma}_{t}$, where ${ }^{\sim}$ denotes a ten-minute moving average, are also shown in Fig. 6(c). The wind vane relative wind direction measurement on the wind turbine nacelle is designed to measure the

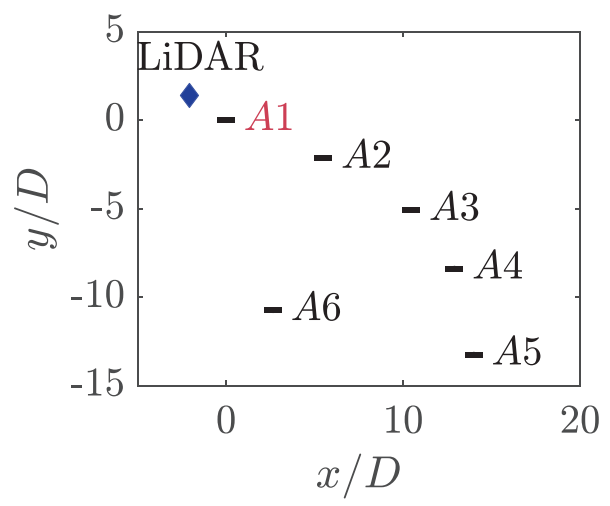

(a)

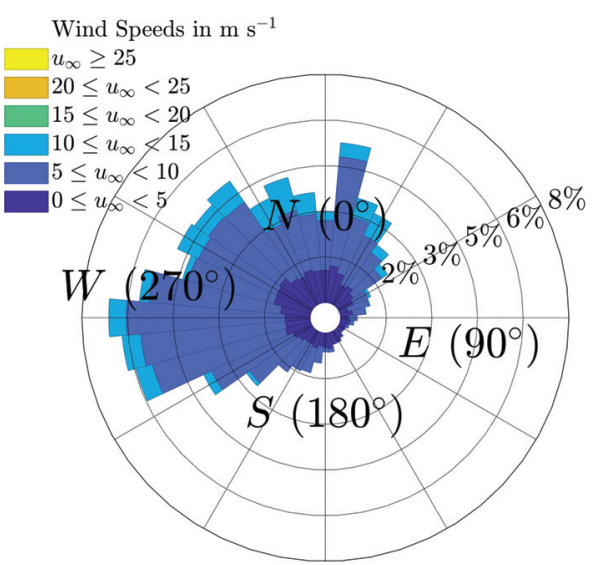

(b)
FIG. 5. (a) Wind turbine cluster $A$ with the corresponding turbine labels. The easting and northing directions, normalized by the wind turbine diameter $D$, are indicated by $x$ and $y$, respectively. Red turbines are actuating turbines with intentional yaw misalignment strategies, and black turbines are yaw aligned. The profiling LiDAR location, approximately $2.5 D$ northwest of turbine $A 1$, is indicated in cluster A. The wind turbines are oriented as if the flow is from the north. (b) Wind rose from the cluster A mast for February and March of 2016 through 2018. 


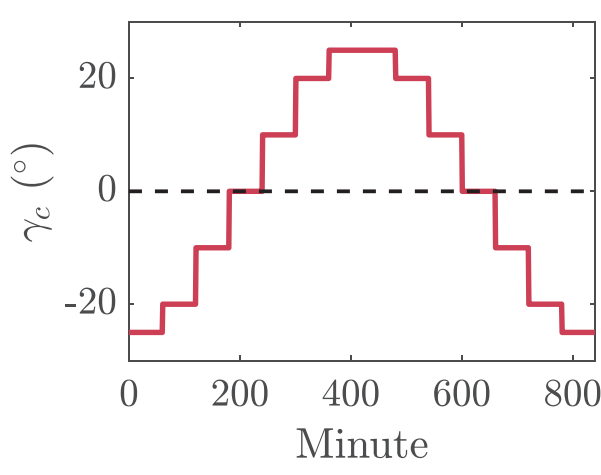

(a)

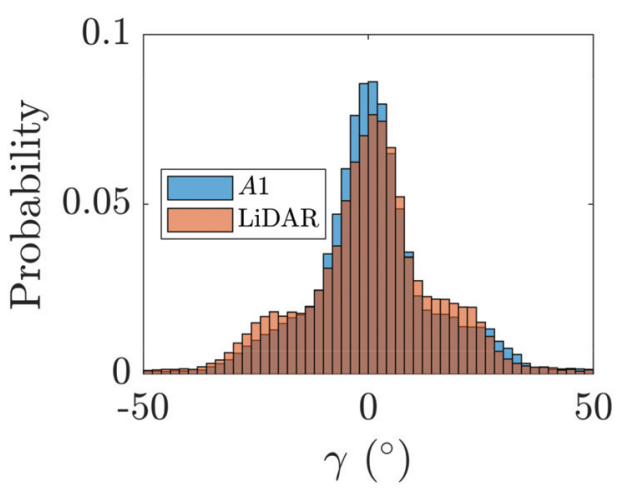

(b)

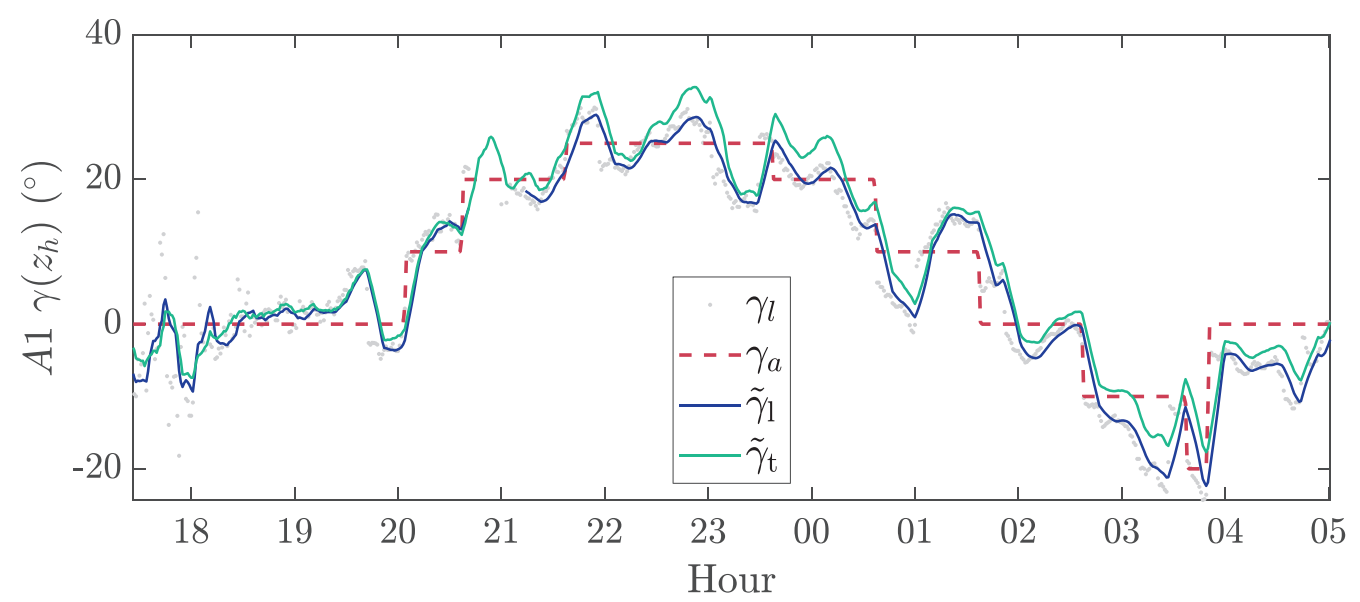

(c)

FIG. 6. (a) Time series of yaw offset $\gamma_{c}$ commands sent to each actuating wind turbine. Each yaw misalignment command is held for one hour, provided that the wind conditions remain within the prescribed threshold parameters. (b) Probability distribution of the yaw misalignment calculated by turbine A1 and the profiling LiDAR. (c) Time series of turbine $A 1$ yaw misalignment field experiment from March 19, 2020. The one-minute averaged yaw misalignment measured by the difference between the turbine nacelle position and the LiDAR wind direction is given by $\gamma_{l}=\alpha_{\text {LiDAR }}\left(z=z_{h}\right)-\beta$, where $\beta$ is the nacelle position, the yaw misalignment applied by the turbine controller is $\gamma_{a}$, the ten-minute moving average of the yaw misalignment measured by the LiDAR is $\tilde{\gamma}_{1}$, and the ten-minute moving average of the yaw misalignment measured by turbine $A 1$ is $\tilde{\gamma}_{t}$.

yaw misalignment offset for relatively low values of yaw. The impact of yaw misalignment on the measurements of the nacelle mounted wind vane is uncertain, and therefore, in this study, the yaw misalignment will be characterized by the LiDAR wind direction and the wind turbine nacelle position $\gamma_{l}$.

\section{A. LiDAR measurements and stability quantification}

In order to establish a qualitative sense of the stability during the experiment, the bulk Richardson number is used, ${ }^{26,64}$

$$
R i_{B}=\frac{\left(g / \bar{\theta}_{T}\right) \Delta \theta_{T} \Delta z}{(\Delta u)^{2}+(\Delta v)^{2}},
$$

where $g$ is the gravitational acceleration and $\theta_{T}$ is the potential temperature. The bulk Richardson number is calculated between two points spaced at a vertical separation distance of $\Delta z=z_{2}-z_{1}$, where $z_{2}>z_{1}$ and the velocity and potential temperature differences between the two heights are $\Delta u, \Delta v$, and $\Delta \theta_{T}$, respectively. The flow is statically unstable when $R i_{B}<0$ and stable when $R i_{B}>0$. The magnitude of the bulk Richardson number indicates a qualitative sense of the dynamic stability of the flow, i.e., the balance between turbulent shear production and suppression of turbulence by stable stratification. Critical values are not precisely defined as they are for the flux Richardson number, and therefore, turbulence is expected even with $R i_{B} \approx 10$ (see discussion in the study by Stull ${ }^{26}$ ). The bulk Richardson number is computed using LiDAR measured velocity recorded at the wind turbine hub height, $z \approx 100 \mathrm{~m}$ and at $z=43 \mathrm{~m}$. Temperature is measured at the ground by a LiDAR and at the 100-m hub height using nacelle-mounted thermometer. Since the velocity and temperature measurements are not collocated and the $\Delta z$ layer is relatively thick compared to best practices, ${ }^{26}$ the bulk Richardson number computed in this study will only be used as a qualitative sense of stability.

The histograms of the bulk Richardson number for hours 4 and 14 of the day during the experiment are shown in Figs. 7(a) and 7(b). 


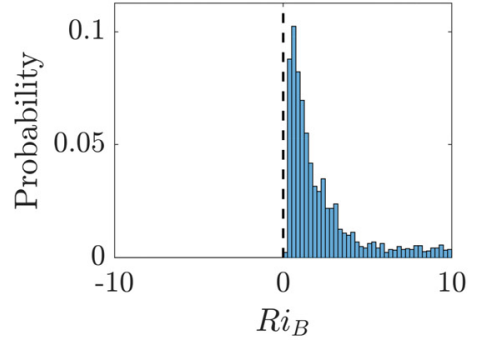

(a)

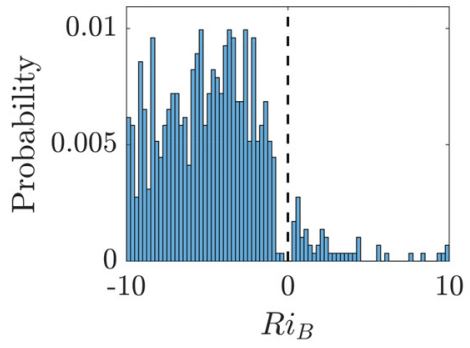

(b)

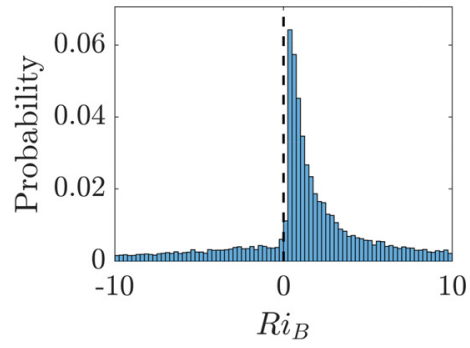

(c)

FIG. 7. Histograms of the bulk Richardson number $R i_{B}$ for hour (a) 4 and (b) 14 and (c) for the flow of interest incident from $-30^{\circ}<\alpha<45^{\circ}$ for any hour. With $R i_{B}<0$, the flow is statically unstable, and with $R i_{B}>0$, the flow is statically stable. $R i_{B}=0$ is shown with a vertical dashed black line. The same bin widths are used for each histogram.

In the early morning (hour 4), the ABL is statically stable with only positive bulk Richardson numbers. Conversely, for hour 14, the ABL is generally unstable with $R i_{B}<0$. The flow of interest for the present experiment focuses on flow from the north between $-30^{\circ}<\alpha<45^{\circ}$. For flow constrained between these directions, the probability distribution of the bulk Richardson is shown in Fig. 7(c); the majority of occasions of flow incident from the north results in statically stable $A B L$ conditions $\left(R i_{B}>0\right)$. Since most of the values of $R i_{B}$ for the wind conditions of interest are positive and small, the flow will have shear turbulence production and be dynamically unstable but with a statically stable stratification that acts to suppress turbulent mixing. As discussed in Sec. II A, the stable ABL with reduced turbulent mixing is expected to have stronger veer compared to the Ekman layer and a pronounced sub-geostrophic jet.

Given the expected stable ABL conditions during the experiment, the experimental results will be characterized in conditional averages depending on the magnitude of shear and veer recorded by the profiling LiDAR. The LiDAR measures at 11 setpoints vertically over the wind turbine rotor area. The shear exponent $\alpha_{v}$ [Eq. (23)] is computed through a least squares fit of the 11 points in the rotor area to a power law profile. The median velocity profile for the northern flow experimental conditions for all values of $\alpha_{v}$ is shown in Fig. 8(a). Overlaid on the curve are ten randomly selected one-minute averaged velocity profiles, and the standard deviation about the median value as a function of $z$ is shown. In the median, $\alpha_{v}=0.12$, but the standard deviation is substantial with negative shear occurring well within one standard deviation, indicating that the flow deviates from a power law with non-negligible frequency. When the velocity profiles are filtered such that the least squares error computed $\alpha_{v}<0$, a sub-geostrophic jet emerges in the median profile with some randomly selected profiles exhibiting significant anti-shear above the wind turbine hub height [Fig. 8(b)]. It is worth noting that a power law results in a poor fit to the velocity profiles in Figs. 8(a) and 8(b), and therefore, $\alpha_{v}$ will be used only as a qualitative measure of the direction of shear in the wind profile. It is also evident from Figs. 8(a) and 8(b) that the magnitude, and even the sign, of $\alpha_{v}$ is a function of $z$, which was also shown in an onshore wind farm in the Midwest of the United States. ${ }^{50}$

The median wind directions as functions of height for wind conditions filtered by $-\infty<\alpha_{v}<\infty$ and $\alpha_{v}<0$ are shown in Fig. 8(c). The median wind direction profiles are both increasing as a function of height, which is positive veering associated with Ekman turning (clockwise turning with increasing $z$ ). The veer profiles are also approximately linear as a function of height, confirming the veer selections made in the canonical wind condition model discussed in Sec. II and shown in Fig. 4. When $\alpha_{v}<0$, the veer is significantly enhanced, with the median veer from the turbine bottom blade tip to top blade tip of $\Delta \alpha=\alpha\left(z=z_{h}+R\right)-\alpha\left(z=z_{h}-R\right) \approx 30^{\circ}$ compared to $\Delta \alpha \approx 15^{\circ}$ for the full $\alpha_{\nu}$ range.

The cumulative density function of the veer over the turbine face $\Delta \alpha$ for the wind conditions of interest is shown in Fig. 9(a). Approximately $90 \%$ of the one-minute averaged data samples have a positive veer and $10 \%$ have negative veer (backing), which is similar to

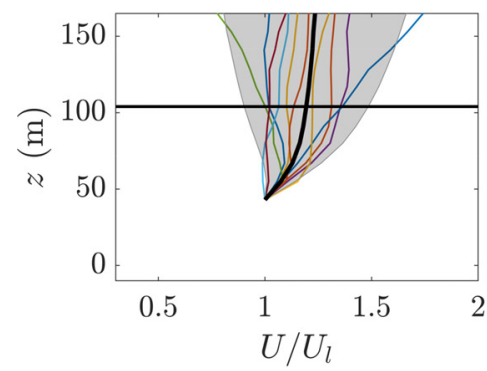

(a)

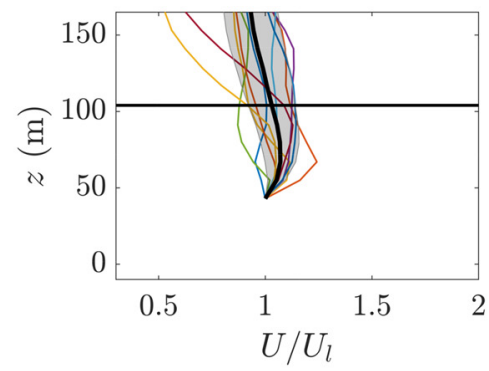

(b)

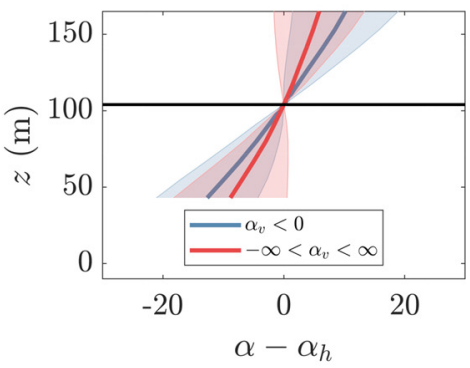

(c)

FIG. 8. Horizontal wind speed $U=\sqrt{u^{2}+v^{2}}$ normalized by the lowest wind speed measured by the profiling LiDAR $U_{1}$ at $z=43 \mathrm{~m}$ above the ground for (a) $-\infty<\alpha_{v}<$ $\infty$ and (b) $\alpha_{v}<0$. The black curve represents the median with the shaded area representing one standard deviation about the median. Colored lines are ten randomly selected one-minute averaged velocity profiles within the wind condition group. The horizontal black line indicates the wind turbine hub height. (c) Median wind direction $\alpha-$ $\alpha_{h}$ for the two directional shear cases in (a) and (b). The shaded error represents one standard deviation in the data. 


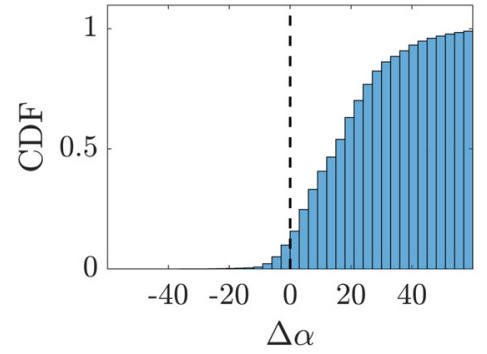

(a)

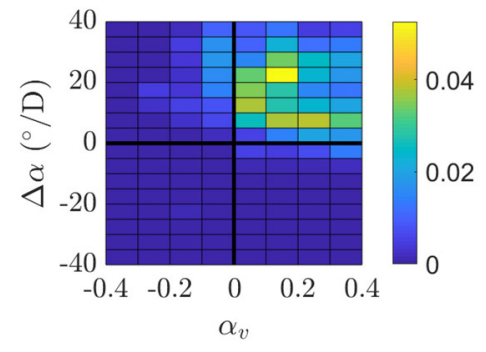

(b)

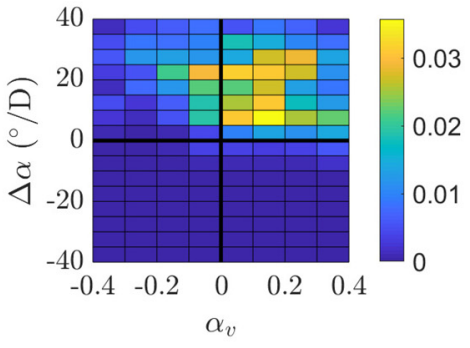

(c)

FIG. 9. (a) Probability distribution of the veer over the wind turbine face $\Delta \alpha$. (b) Joint probability distribution of the shear and veer measured for flow incident from the north. (c) Joint probability distribution of the shear above the hub height and veer measured for flow incident from the north.

other field studies in flat terrain onshore wind farms (e.g., Sanchez Gomez and Lundquist ${ }^{50}$ ). Further, approximately $50 \%$ of the veer cases result in $\Delta \alpha>20^{\circ}$. The joint probability distribution of $\Delta \alpha$ and $\alpha_{v}$ is shown in Fig. 9(b), for $\alpha_{v}$ computed using velocity measurements recorded between 43 and $165 \mathrm{~m}$ above the ground. The majority of one-minute averaged instances occur in quadrant $\mathrm{I}$, with $\alpha_{v}, \Delta \alpha>0$, and the following most frequent is quadrant II, with $\alpha_{v}<0$ and $\Delta \alpha>0$. As also shown in the cumulative distribution function in Fig. 9(a), $\Delta \alpha<0$ occurs infrequently. The shear exponent is also computed considering vertical locations such that $z>z_{h}$ and the joint probability distribution is shown in Fig. 9(c). Comparing Figs. 9(b) and 9(c), the frequency of $\alpha_{v}<0$ has significantly increased, indicating that the velocity profile above the wind turbine hub height often experiences negative shear with respect to the velocity at the wind turbine hub height. Negative shear above the hub height occurs approximately $35 \%$ of the time, and $\alpha_{v}<0.1$ occurs in $53 \%$ of the oneminute averaged samples.

The power available in the incoming wind $P \propto(\vec{u} \cdot \hat{n})^{3}$, where $\vec{u}$ is the incoming wind vector and $\hat{n}$ is the unit vector normal to the wind turbine rotor area. The power available in the incoming wind will, therefore, depend on the specific wind speed and direction profiles. Qualitatively, the probability distributions of $\alpha_{v}$ indicate that the available power in the wind is larger below the hub height than above the hub height with reasonable frequency at the wind farm in northwest India.

\section{RESULTS}

The power ratio $\left[P_{r}=P_{A 1} / P_{A 2}\right.$, Eq. (1)] is calculated for intentionally yaw misaligned turbine $A 1$ with respect to the power production of baseline turbine $A 2$ [see Fig. 5(a) for the farm layout]. Turbine $A 2$ is a turbine directly adjacent to $A 1$ for wind incident from the north or northeast, and the profiling LiDAR provides controlled wind measurements. The influence of yaw misalignment on power production is computed as $P_{r}=P_{A 1} / P_{A 2}$ instead of $P_{r}=P_{A 1}(\gamma) / P_{A 1}(\gamma=0)$, to ensure that the same incident wind profile is encountered by the yaw misaligned and aligned turbines.

The wind conditions for cluster $A$ are restricted such that the wind direction at the wind turbine hub height is $-30^{\circ}<\alpha<45^{\circ}$ to ensure that there is no waked inflow from potential upwind turbines outside the wind direction band of interest, as discussed in Sec. III. Additional quality filters are in place in the SCADA data to ensure that the wind turbines are operating normally with no power limitations, such as grid curtailment, and the yaw control system is active. The turbulence intensity is constrained below $T I<10 \%$ to reduce the variability in the wind conditions incident to turbines $A 1$ and $A 2$ although the results are similar with this constraint relaxed.

Given the experimental window of almost two months and the wind condition and data quality filters, 8,376 unique, one-minute averaged data samples were collected, which amount to nearly 6 full days of yaw misalignment actuation spread over the two-month period. This results in approximately 700 unique data points within each yaw misalignment offset command [Fig. 6(a)]. As shown in Fig. 6(c), due to the underlying dynamics of the native yaw control system, there are some deviations between the intended yaw misalignment and the realized yaw misalignment, as computed by the difference between the LiDAR wind direction at the hub height and the wind turbine nacelle position. The experimental $P_{r}$ results will, therefore, be analyzed with respect to the realized one-minute averaged yaw misalignment value, $\gamma_{l}=\alpha_{\mathrm{LiDAR}}\left(z=z_{h}\right)-\beta$ [Eq. (9)] rather than the SCADA applied yaw value. The mean Taylor's hypothesis advection time between the LiDAR and the wind turbines of interest is less than one minute. The advection time lag is not included in the following analysis, but the results are similar with an advection lag incorporated. Given the form of $P_{r}$ and that the wind speeds are restricted to Region II of the power curve, the particular value of the incident wind speed does not significantly influence $P_{r}$.

The power ratio $P_{r}$ for the full experimental dataset is shown in Fig. 10(a). There are 8376 unique one-minute averaged data samples in the full experimental dataset after the quality filters described above. The realized yaw misalignment values $\gamma_{l}$ are binned in $1^{\circ}$ increments, and the data within the middle $80 \%$ of the probability distribution for each yaw misalignment bin are shown to alleviate the influence of one-minute averaged outliers. The median of the middle $80 \%$ is also shown with one standard deviation about the median representing the error bars. Reference curves for $\cos ^{2}(\gamma)$ and $\cos ^{3}(\gamma)$ are also shown. Finally, the model presented in Sec. II [Eq. (19)] is computed given the incident wind speed and direction profiles measured by the LiDAR for each one-minute average sample. The torque controller generator torque is prescribed as $T_{c}=K \Omega^{2}$. The median and standard deviation about the median for the model are also shown in Fig. 10.

For the full experimental dataset [Fig. 10(a)], the power ratio approximately follows $\cos ^{2}(\gamma)$ although $\cos ^{3}(\gamma)$ is generally within one standard deviation of the data. $P_{r}$ is asymmetric, with $P_{r}\left(\gamma_{l}>0\right)$ $>\operatorname{Pr}\left(\gamma_{l}<0\right)$ for a fixed absolute value of $\gamma_{l}$. With $\gamma_{l}>0, \cos ^{2}(\gamma)$ is 


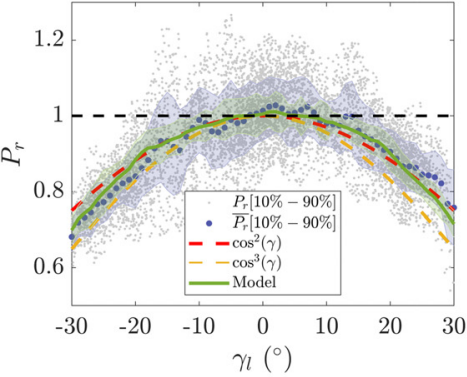

(a)

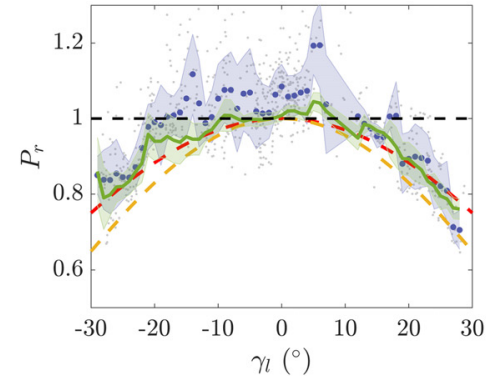

(b)

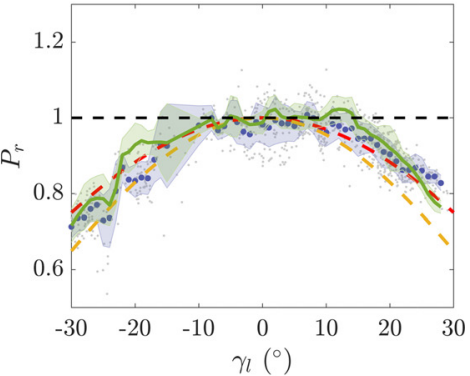

(c)

FIG. 10. Blade element model comparison. Power ratio $P_{r}=P_{A 1} / P_{A 2}$ for incident wind directions of $-30^{\circ}<\alpha\left(z=z_{h}\right)<45^{\circ}$ as measured by the LiDAR. The yaw misalignment values are binned in $1^{\circ}$ increments. Within each yaw misalignment bin, $10 \%$ tails on the upper and lower ends of the $P_{r}$ PDF are removed. $\bar{P}_{r}[10 \%-90 \%]$ denotes the median of the central $80 \%$ of the $P_{r}$ PDF. The yaw misalignment $\gamma_{l}$ is calculated by the LiDAR $\gamma_{1}=\alpha_{L \text { LDAR }}-\beta_{A 1}$. (a) All conditions of shear and veer are considered, and the turbulence intensity is constrained by $\mathrm{TI}<10 \%$. The number of resulting data points is $n=8376$. (b) $\alpha_{v}>0.2, \Delta \alpha_{v}>20^{\circ}, n=873$ and (c) $\alpha_{v}<0, \Delta \alpha_{v}>20^{\circ}$, $n=996$. Conditional bins with more than five data points are shown.

an underestimate of $P_{r}$ but always remains within one standard deviation of the median value (hereafter, an underestimate indicates that the model predicts a value of $P_{r}$ that is less than the experimental value, indicating that the power loss due to yaw misalignment is overestimated). The curve for $\cos ^{3}(\gamma)$ is also within one standard deviation of the median for $\gamma_{l}>0$ except for high values of $\gamma_{l}>25^{\circ}$. On the contrary, for $\gamma_{l}<0, \cos ^{2}(\gamma)$ is an overestimate of $P_{r}$ (the experimentally measured $P_{r}$ is lower than the $\cos ^{2}(\gamma)$ estimate) and falls outside of one standard deviation around the median for $\gamma_{l}<-25^{\circ}$. For $\gamma_{l}<0, \cos ^{3}(\gamma)$ is always within one standard deviation of the median. These results reflect the expectation that $P_{r}$ will be asymmetric about $\gamma_{l}=0$ for spatially heterogeneous flow conditions in $z$. The model generally follows $\cos ^{2}(\gamma)$ with a slight deviation and asymmetry present; the model predicts that $\gamma_{l}>0$ produces slightly higher values of $P_{r}$ than $\gamma_{l}<0$ as the data also represent.

In order to account for potential causes of the asymmetry in $P_{r}$ as a function of $\gamma_{b}$, we will introduce wind condition restrictions on the full, recorded dataset. Two distinct wind condition restrictions are introduced, with each containing approximately $10 \%$ of the experimental dataset. First, positive shear and veer conditions are considered with $\alpha_{v}>0.2$ and $\Delta \alpha>20^{\circ}$; this condition contains 873 unique oneminute averaged data samples and is associated with power-law-type velocity profiles. Second, negative shear and positive veer conditions are considered with $\alpha_{v}<0$ and $\Delta \alpha>20^{\circ}$; this condition contains 996 unique one-minute averaged data samples. The second condition is associated with a stable boundary layer, Ekman-type flows exhibiting sub-geostrophic jets. Additionally, as shown in Fig. 8(c), the negative shear condition will generally be associated with larger veer magnitude than positive shear.

In Fig. $10(\mathrm{~b}), \alpha_{v}>0.2$ and $\Delta \alpha>20^{\circ}$, and there is a significant modification to the $P_{r}$ results. In particular, there is a significant increase in $P_{r}\left(\gamma_{l}<0\right)$ and a moderate reduction in $P_{r}\left(\gamma_{l}>0\right)$. Within these conditions, $\cos ^{2}(\gamma)$ is an underestimate of $P_{r}\left(\gamma_{l}<0\right)$, compared to the previous results considering all $\alpha_{v}$ where $\cos ^{2}(\gamma)$ overestimated $P_{r}\left(\gamma_{l}<0\right)$. With a positive veering angle associated with clockwise Ekman spiraling, a negative hub height yaw misalignment results in a smaller relative local yaw misalignment angle [Eq. (11)] above the hub height than below the hub height. With a strong positive shear exponent, $\alpha_{v}>0.2$, the wind speed also increases as a function of $z$. Therefore, the local available power $(\vec{u} \cdot \hat{n})^{3}$ will be larger for a hub height yaw misalignment of $\gamma<0$ than for $\gamma>0$. The model proposed in this study is able to capture the qualitative trend observed in the data where $P_{r}\left(\gamma_{l}<0\right)>P_{r}\left(\gamma_{l}>0\right)$.

In Fig. 10 (c), the wind conditions are restricted to $\Delta \alpha>20^{\circ}$ and $\alpha_{v}<0$. Given these wind conditions, there are an increase in $P_{r}\left(\gamma_{l}>0\right)$ and a reduction in $P_{r}\left(\gamma_{l}<0\right)$. For negative shearing conditions, there is, in general, more energy below the wind turbine hub height of $z=z_{h}$ than above it. Again, given $\Delta \alpha>20^{\circ}$, a positive yaw misalignment angle will locally align the rotor area with the inflow below the hub height, and therefore, $P_{r}\left(\gamma_{l}>0\right)>P_{r}\left(\gamma_{l}<0\right)$ is expected. Further, since the veering angle is more significant in negative shearing conditions [as discussed in Sec. II A and shown in Fig. 8(c)], the reduction in $P_{r}$ for $\gamma_{l}<0$ is expected to be more substantial than the reduction in $P_{r}$ for $\gamma_{l}>0$ when $\alpha_{v}>0$. In Fig. 10(c), there are sharp reductions in $P_{r}$ for certain instances of $\gamma_{l}<0$, confirming this expectation. Again, the model captures the qualitative trend in $P_{r}$ although some quantitative discrepancies exist.

There are a few potential sources of discrepancy between the model presented in Sec. II and the field experimental data. There is uncertainty associated with the impact of yaw misalignment on the measurements of the wind turbine nacelle-mounted wind speed and direction sensors. ${ }^{65}$ These measurements, in turn, dictate the turbine control system operational state. Further, there is uncertainty associated with the wind direction calibrations (such that $0^{\circ}$ corresponds to true north) for the yaw actuating and yaw aligned turbines, as well as the profiling LiDAR. This uncertainty is estimated to be approximately $\pm 1^{\circ}$ for each device. For the model, higher order aeroelastic effects on the blades may modify the incident angle of attack $\phi-\psi$ in Eqs. (13) and (14), which could correspondingly modify the solution for $\Omega(\gamma)$. Variations in axial induction over the rotor area were not considered, as the applicability of these empirical corrections for sheared and veered conditions is uncertain and could be examined in future work. The quasi-static model assumes that the aerodynamic and generator torques are in equilibrium, which may not always hold for a given one-minute average due to the underlying dynamics of the generator torque control system. Finally, the blade element model captures oneminute averaged variations in wind speeds, but higher frequency or intermittent incident wind content, such as wind gusts, which have a 


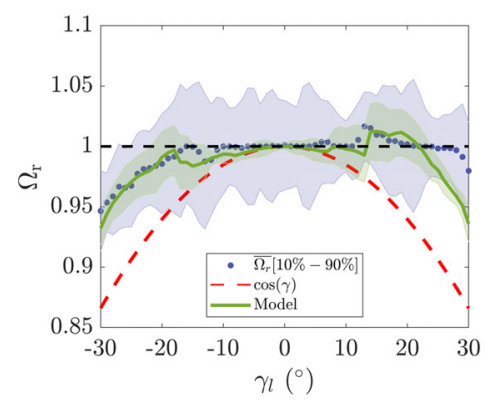

(a)

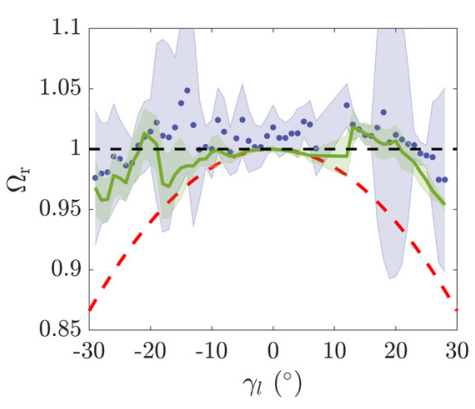

(b)

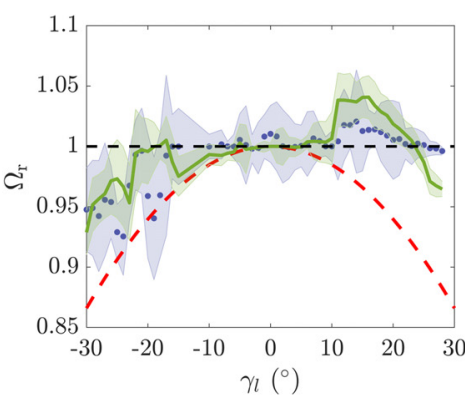

(c)

FIG. 11. Blade element model comparison. Angular velocity ratio $\Omega_{r}=\Omega_{A 1} / \Omega_{A 2}$ for incident wind directions of $-30^{\circ}<\alpha\left(z=z_{h}\right)<45^{\circ}$ as measured by the LiDAR. The yaw misalignment values are binned in $1^{\circ}$ increments. Within each yaw misalignment bin, $10 \%$ tails on the upper and lower ends of the $\Omega_{r}$ PDF are removed. $\bar{\Omega}_{r}[10 \%-90 \%]$ denotes the median of the central $80 \%$ of the $\Omega_{r}$ PDF. The yaw misalignment $\gamma_{1}$ is calculated by the LiDAR $\gamma_{1}=\alpha_{\text {LiDAR }}-\beta_{A 1}$. (a) All conditions of shear and veer are considered, and the turbulence intensity is constrained by $\mathrm{TI}<10 \%$. The number of resulting data points is $n=8376 .(\mathrm{b}) \alpha_{v}>0.2, \Delta \alpha_{v}>20^{\circ}, n=873$ and (c) $\alpha_{v}<0, \Delta \alpha_{v}>20^{\circ}, n=996$. Conditional bins with more than five data points are shown.

nonlinear impact on the power, torque, and angular velocity were not considered. The angular velocity ratios $\Omega_{r}=\Omega(\gamma) / \Omega(\gamma=0)$ for the three wind conditions are shown in Fig. 11. While the model is able to predict the qualitative trends of $\Omega(\gamma)$, there are quantitative discrepancies, especially for $\gamma>0$. In order to alleviate these issues while maintaining the analytical nature of the model presented in Sec. II, we perform a semi-empirical model calculation where the resulting value of $\Omega$ is used to predict $T_{r}$ and $P_{r}$. While this method will not be available in a practical application setting since it requires a field experiment to measure $\Omega_{\gamma}$, this will serve as a validation of the model for the prediction of $T_{r}$ and $P_{r}$, which are not a trivial result of $\Omega_{\gamma}$ (see $T_{r}$ and $P_{r}$ derivation in Sec. II). The model results for $T_{r}$ and $P_{r}$ are given in Figs. 12 and 13, respectively.

The mean absolute errors of the predicted $P_{r}$ associated with various cosine models, the physics-based blade element model, and the semi-empirical blade element model are shown in Fig. 14. The semiempirical model has the lowest mean absolute error for all wind condition cases. $\cos ^{2}\left(\gamma_{l}\right)$ achieves the lowest error of the cosine models except for $\alpha_{v}>0.2$ data. The physics-based model has lower error than all cosine model approximations for all cases except for the $\cos ^{2}\left(\gamma_{l}\right)$ model for the negative shear dataset, highlighting the asymmetric, complex influence of the incident wind conditions. It is worth noting that there is not a precise physical justification for the form of the cosine model [Eq. (1)] or the associated value of the $P_{p}$ exponential factor (see, e.g., discussion by Pederson ${ }^{65}$ or Bastankhah and Porté-Agel ${ }^{58}$ ), and therefore, the application of the correct $P_{p}$ to reduce the power ratio prediction error is unknown a priori, while the physics-based blade element model is fully predictive. The quantitative agreement between the field data and the model presented in Sec. II is significantly improved in the semi-empirical formulation, with the model capturing sharp, nonmonotonic trends present in the field data with reasonable accuracy. The success of the aerodynamic model presented in Sec. II for qualitative predictions of $P_{r}$ without $\Omega_{\gamma}$ and improved quantitative predictions with $\Omega_{\gamma}$ suggests that the model can be used before wake steering control to estimate $P_{r}$ given the aerodynamic properties of the turbine of interest.

In order to further detail the asymmetric trends of the measured $P_{r}(\gamma)$ and the model, the normalized difference between the power ratio for positive and negative yaw misalignment is computed,

$$
\Delta P_{r}=\frac{P_{r+}-P_{r-}}{\frac{1}{2}\left(P_{r+}+P_{r-}\right)},
$$

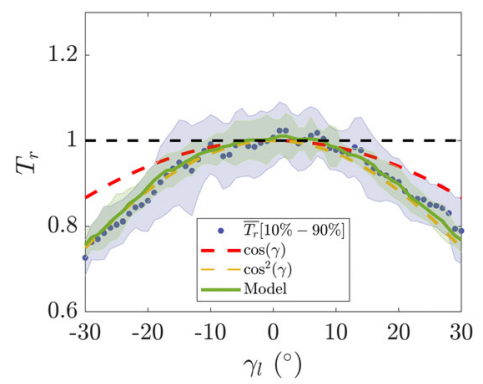

(a)

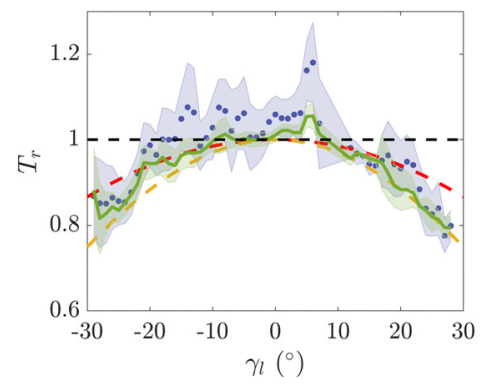

(b)

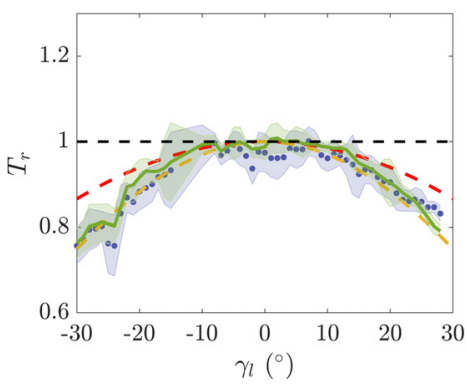

(c)

FIG. 12. Semi-empirical blade element model comparison. Torque ratio $T_{r}=T_{A 1} / T_{A 2}$ for incident wind directions of $-30^{\circ}<\alpha\left(z=z_{h}\right)<45^{\circ}$ as measured by the LiDAR. The yaw misalignment values are binned in $1^{\circ}$ increments. Within each yaw misalignment bin, $10 \%$ tails on the upper and lower ends of the $T_{r}$ PDF are removed. $\bar{T}_{r}[10 \%-90 \%]$ denotes the median of the central $80 \%$ of the $T_{r}$ PDF. The yaw misalignment $\gamma_{l}$ is calculated by the LiDAR $\gamma_{I}=\alpha_{L \text { LDAR }}-\beta_{A 1}$. (a) All conditions of shear and veer are considered, and the turbulence intensity is constrained by $\mathrm{TI}<10 \%$. The number of resulting data points is $n=8376$. (b) $\alpha_{v}>0.2, \Delta \alpha_{v}>20^{\circ}, n=873$ and (c) $\alpha_{v}<0, \Delta \alpha_{v}>20^{\circ}, n=996$. Conditional bins with more than five data points are shown. 


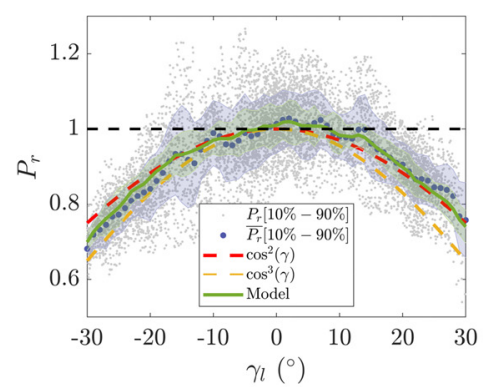

(a)

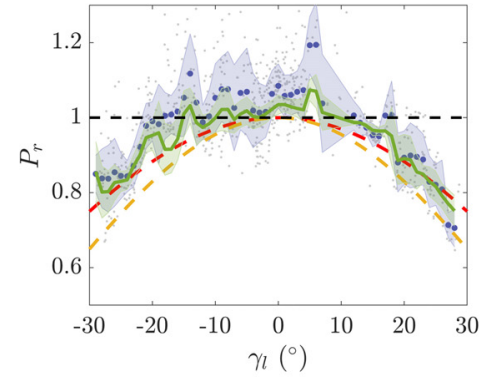

(b)

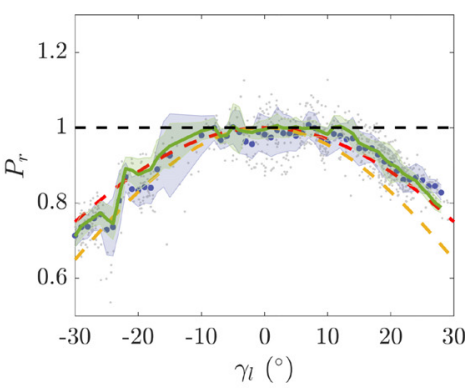

(c)

FIG. 13. Semi-empirical blade element model comparison. Power ratio $P_{r}=P_{A 1} / P_{A 2}$ for incident wind directions of $-30^{\circ}<\alpha\left(z=z_{h}\right)<45^{\circ}$ as measured by the LiDAR. The yaw misalignment values are binned in $1^{\circ}$ increments. Within each yaw misalignment bin, $10 \%$ tails on the upper and lower ends of the $P_{r}$ PDF are removed. $\bar{P}_{r}[10 \%-90 \%]$ denotes the median of the central $80 \%$ of the $P_{r}$ PDF. The yaw misalignment $\gamma_{1}$ is calculated by the LiDAR $\gamma_{1}=\alpha_{L \text { LDAR }}-\beta_{A 1}$. (a) All conditions of shear and veer are considered, and the turbulence intensity is constrained by $\mathrm{TI}<10 \%$. The number of resulting data points is $n=8376$. (b) $\alpha_{v}>0.2, \Delta \alpha_{v}>20^{\circ}, n=873$ and (c) $\alpha_{v}<0, \Delta \alpha_{v}>20^{\circ}, n=996$. Conditional bins with more than five data points are shown.

where $P_{r+}$ indicates $P_{r}\left(\gamma_{l}>0\right)$ and $P_{r-}$ indicates $P_{r}\left(\gamma_{l}<0\right)$. For cosine models of the power ratio [Eq. (1)], $\Delta P_{r}=0 \forall \gamma$ by definition. The profiles of $\Delta P_{r}$ for $-\infty<\Delta \alpha<\infty$ and $-\infty<\alpha_{v}<\infty, \Delta \alpha$ $>20^{\circ}$ and $\alpha_{v}>0.2$, and $\Delta \alpha>20^{\circ}$ and $\alpha_{v}<0$ are shown in Fig. 15 for the experimental data and the physics-based model. The full dataset exhibits an asymmetry such that $P_{r}\left(\gamma_{l}>0\right)>\operatorname{Pr}\left(\gamma_{l}<0\right)$. The model predicts a slightly higher value for $P_{r}\left(\gamma_{l}>0\right)$ and is within one standard deviation of the experimental data, but the quantitative agreement is not precise. For restricted positive or negative values of $\alpha_{v}$, as shown in Figs. 15(b) and 15(c), the model reproduces the qualitative trend observed in the field data as well as an improved quantitative accuracy. Interestingly, there are occasional discrete modulations in $\Delta P_{r}\left(\gamma_{l}\right)$ that result in a nonmonotonic profile as a function of $\gamma_{l}$. Since the model, in general, quantitatively captures these discrete events, there are two likely explanations for this nonmonotonic behavior that act in tandem. Given the strong veering and shearing conditions observed during the experiment, the hub height yaw misalignment angle that produces maximum power is not necessarily zero, as also discussed by Kragh and Hansen ${ }^{54}$ with respect to shear and Murphy et al. ${ }^{49}$ with respect to shear and veer. Therefore, the peak $P_{r}$ may not occur at $\gamma_{l}=0$. Further, even with the wind condition filters on $\alpha_{v}$ and $\Delta \alpha$, a variety of wind conditions are realized due to the complex nature of the turbulent $\mathrm{ABL}$ flow in a field

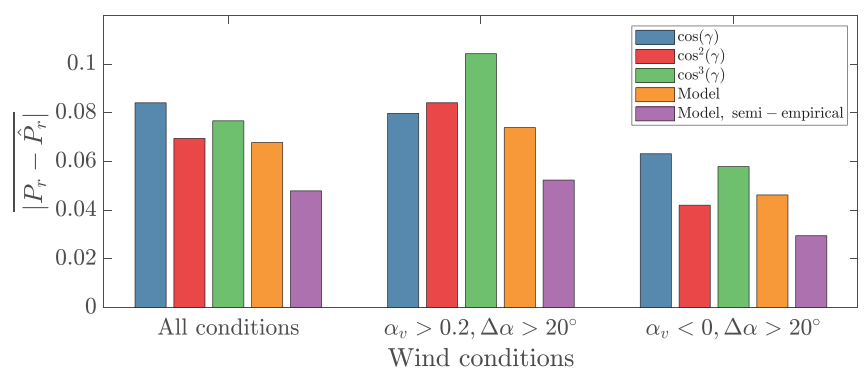

FIG. 14. Mean absolute error between the measured power ratio $P_{r}$ and the predicted power ratio $\hat{P}_{r}$ for various cosine models, the predictive physics-based blade element model, and the semi-empirical blade element model where the model is provided $\Omega_{r}$. environment (see also randomly selected velocity profiles in Fig. 8). Given the variety of velocity and direction profiles realized within the wind condition bins, the trends in $P_{r}$ not only are isolated to $\gamma_{l}$ but also have a functional dependence on the wind conditions themselves. Since the model resolves the leading-order effects of these variations in $u(z)$ and $\alpha(z)$, the model captures these discrete events with reasonable accuracy.

As with $P_{r}$, we can also compute $\Delta P_{r}$ using the semi-empirical approach wherein the model is provided $\Omega_{\gamma}$. The asymmetry of the power ratio $\Delta P_{r}$ for the semi-empirical model is shown in Fig. 16, where the qualitative and quantitative experimental results are reproduced within the errorbars of the field data for nearly all data-points.

\section{DISCUSSION AND IMPLICATIONS FOR WAKE STEERING CONTROL}

The full-scale field experimental results presented in Sec. IV confirm the expectation that wind turbines in yaw misalignment will exhibit asymmetric power production as a function of the sign of the yaw misalignment angle depending on the incident wind conditions. For wind velocity profiles that follow a power law with a positive shear exponent and exhibit clockwise Ekman turning associated with Coriolis forces in the northern hemisphere, negative yaw misalignment leads to enhanced power production for the yawed turbine compared to positive yaw misalignment. On the other hand, for strongly stable conditions where positive veering and a sub-geostrophic jet emerge, positive yaw misalignment is beneficial compared to negative yaw misalignment. The asymmetric influence of the wind conditions on the power production of a yaw misaligned turbine is represented with the model proposed in Sec. II B. While the quantitative value of $P_{r}$, and asymmetry of $P_{r}$ as a function of the direction of the yaw misalignment, will depend on the wind turbine control system and local wind conditions, the simple model proposed in Sec. II B predicts $P_{r}(\gamma)$ with reasonable accuracy, suggesting that the model can be used to estimate $P_{r}(\gamma)$ for wind turbines without requiring months long field experiments. Further improvements in the predictive capabilities of the model are expected if the influence of the yaw misalignment on the measurements of the nacelle-mounted turbine sensors, and therefore the torque controller, is quantified. The quantitative predictions of the power ratio may also be improved if full aeroelastic solvers that 


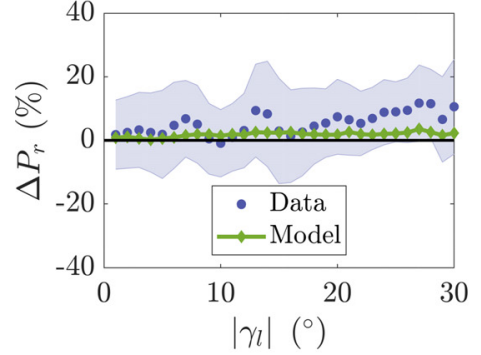

(a)

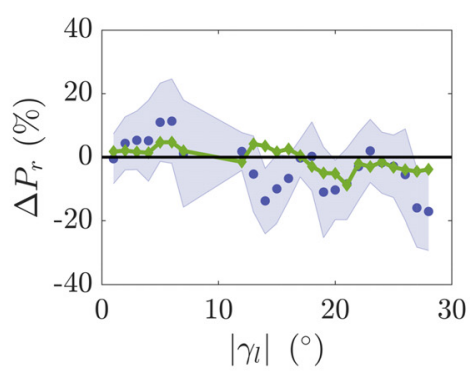

(b)

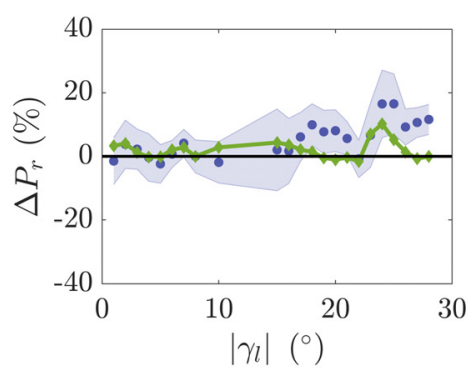

(c)

FIG. 15. Physics only model. The difference in the power ratio between positive yaw misalignment $\left(P_{r+}\right)$ and negative yaw misalignment $\left(P_{r-}\right)$ with a fixed absolute value computed as $\Delta P_{r}=2\left(P_{r+}-P_{r-}\right) /\left(P_{r+}+P_{r-}\right)$ : (a) all shear and veer cases and for $\Delta \alpha>20^{\circ}$, (b) $\alpha_{v}>0.2$, and (c) $\alpha_{v}<0$. Conditional bins with more than five data points are shown.

incorporate the effects of shear, veer, yaw misalignment, and turbine torque control are used. However, the simple model proposed in Sec. II captures the trends of the complex field data, highlighting the impact of the influence of wind shear and veer on the power production of wind turbines in yaw misalignment.

As discussed in Sec. I, previous simulations and field experiments have shown a potential asymmetry in the power production of a wake steering scenario based on the direction of the yaw misalignment for fixed magnitudes of yaw. The asymmetries in $P_{r}, T_{r}$, and $\Omega_{r}$ found in this field experiment and modeled in Sec. II represent another potential cause of asymmetry in the wind turbine array power production given wake steering control as a function of the sign of $\gamma$, aside from the curled wake, ${ }^{31,66}$ Coriolis effects, ${ }^{28}$ or the wake rotation direction. ${ }^{3}$ Importantly, the asymmetry in the success of wake steering as a function of yaw misalignment is case specific and the turbine array power production is not always higher given $\gamma>0$ than $\gamma<0$, as this asymmetry depends on the alignment of the wind turbines and the wind conditions. The same magnitude and direction of asymmetry have not been observed in all studies. Since the asymmetry in the power, torque, and angular velocity ratios of the upwind, yaw misaligned turbine depends on the wind conditions, the asymmetry in the total wind farm power given a wake steering strategy is also expected to depend on the characteristics, and in particular, the stability, of the ABL.

When maximizing wind farm power production using wake steering, the optimal yaw misalignment angles, as well as the resulting power production increase, depend strongly on the power ratio. Recent simulations have shown that for an incorrect estimate of $P_{p}$ in the simple power ratio model $P_{r}=\cos ^{P_{p}}(\gamma)$, the power production for the wind farm can be reduced by wake steering compared to standard individual turbine control. ${ }^{25}$ The results of this field experiment suggest that the standard, symmetric $P_{p}$ model is insufficient and will lead to asymmetric and site- and time-dependent errors in $P_{r}$. Instead, the siteand time-specific wind speed and direction profiles, measured using MET masts or LiDARs, should be leveraged to correct the $P_{r}$ model. Future work should investigate the potential for ground-based extrapolation methods to provide the wind conditions (e.g., Lackner et al. ${ }^{67}$ ) in the absence of LiDAR or MET mast wind profile measurements.

While forces on the wind turbine were not measured in the field experiment, the asymmetric behavior of the power production is also expected in the axial force [Eq. (15)]. Future work should investigate the joint influence of shear, veer, and yaw misalignment on the blade bending moments, which are influenced by yaw misalignment. ${ }^{68}$

\section{CONCLUSIONS}

A field experiment was performed at a wind farm in northwest India involving multiple utility-scale wind turbines. The power production of a freestream wind turbine in yaw is asymmetric depending on the direction of the yaw misalignment. The asymmetry in the power as a function of yaw is chiefly caused by the incident wind speed and direction profiles, the direction of the wind turbine blade rotation,

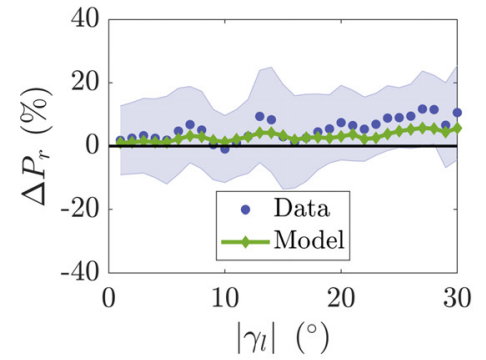

(a)

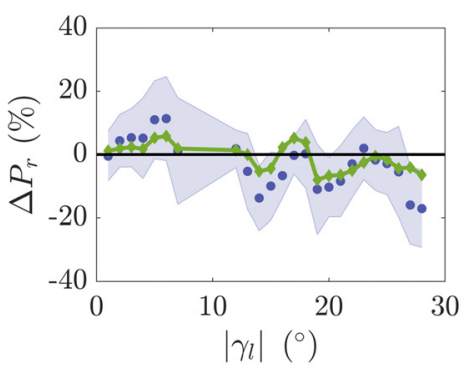

(b)

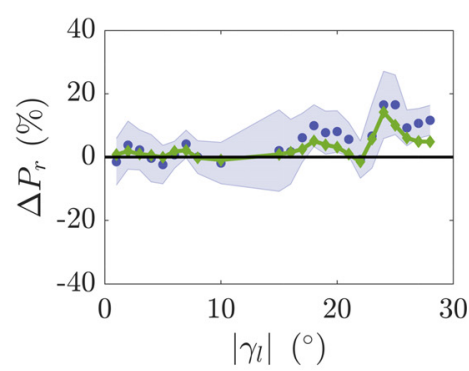

(c)

FIG. 16. Semi-empirical model. The difference in the power ratio between positive yaw misalignment $\left(P_{r+}\right)$ and negative yaw misalignment $\left(P_{r-}\right)$ with a fixed absolute value computed as $\Delta P_{r}=2\left(P_{r+}-P_{r-}\right) /\left(P_{r+}+P_{r-}\right)$ : (a) all shear and veer cases and for $\Delta \alpha>20^{\circ}$, (b) $\alpha_{v}>0.2$, and (c) $\alpha_{v}<0$. Conditional bins with more than five data points are shown. 
the turbine control system, and potential asymmetric effects on turbine sensor systems. Therefore, for differing incident wind conditions during a typical diurnal atmospheric boundary layer evolution, the power production of a freestream turbine as a function of yaw, and its associated asymmetry, may be modified.

The angular velocity of a variable speed wind turbine that uses a generator torque control system does not follow $\cos (\gamma)$ and, instead, depends jointly on the yaw misalignment and incident wind conditions. The angular velocity $\Omega(\gamma)$ is a consequence of the generator torque control system and was persistently larger than $\Omega(\gamma=0) \cdot \cos (\gamma)$ for the yaw misaligned turbine of interest in this study.

A model for the prediction of the power of a yaw misaligned turbine for arbitrary inflow wind conditions and turbine geometry was developed. Previous model approaches predict that the power of a yaw misaligned turbine operating in freestream conditions $P(\gamma)$ $\sim \cos ^{3}(\gamma)$, which differs from experimental measurements. The prediction of $P(\gamma) \sim \cos ^{3}(\gamma)$ directly follows from an assumption that $\Omega(\gamma)=\Omega(\gamma=0) \cdot \cos (\gamma)$; this assumption was found to be inaccurate for a generator torque controlled variable speed turbine. The current model, which calculates the angular velocity as a function of the generator torque control system, aerodynamic forces, yaw misalignment, and wind velocity and direction profiles, predicts that $P(\gamma) \approx P(\gamma=0) \cos ^{2}(\gamma)$ for the presently studied turbine, with asymmetric deviations caused by the incident wind conditions. It is important to note that the specific scaling predicted by the model, and achieved in practice by the wind turbine, will depend on the turbine generator torque controller and the incident wind conditions at the wind farm site, but, in general, the yaw misaligned power will not follow $\cos ^{3}(\gamma)$ unless the angular velocity follows $\cos (\gamma)$. This reaffirms the expectation that the power of a yaw misaligned turbine is not only turbine model-specific but also site-specific. Future wake steering applications can leverage the model presented in this study to compute an expected power ratio $P_{r}$ for a given wind turbine of interest before field or computational deployments. The simple model proposed in Sec. II can be used with arbitrary wind turbines and inflow profiles and can be coupled with dynamic wake models to estimate the power ratio for yawed wind turbines operating in the wakes of upwind turbines. Future work should investigate the optimal generator torque control strategy to minimize the power production degradation as a function of the yaw misalignment depending on the incident wind conditions.

\section{ACKNOWLEDGMENTS}

M.F.H. is funded through a National Science Foundation Graduate Research Fellowship under Grant No. DGE-1656518 and a Stanford Graduate Fellowship. The authors would like to thank Sanjiva Lele for thoughtful suggestions on the blade element model and Varun Sivaram for support throughout this study.

\section{DATA AVAILABILITY}

The data used in this study are confidential at the request of the wind farm operator.

\section{REFERENCES}

${ }^{1}$ A. C. Kheirabadi and R. Nagamune, "A quantitative review of wind farm control with the objective of wind farm power maximization," J. Wind Eng. Ind. Aerodyn. 192, 45-73 (2019).
${ }^{2}$ P. Fleming, P. M. Gebraad, S. Lee, J.-W. van Wingerden, K. Johnson, M. Churchfield, J. Michalakes, P. Spalart, and P. Moriarty, "Simulation comparison of wake mitigation control strategies for a two-turbine case," Wind Energy 18, 2135-2143 (2015).

${ }^{3}$ P. Gebraad, F. Teeuwisse, J. Van Wingerden, P. A. Fleming, S. Ruben, J. Marden, and L. Pao, "Wind plant power optimization through yaw control using a parametric model for wake effects-a CFD simulation study," Wind Energy 19, 95-114 (2016).

${ }^{4}$ F. Campagnolo, V. Petrović, C. L. Bottasso, and A. Croce, "Wind tunnel testing of wake control strategies," in 2016 American Control Conference (ACC) (IEEE, 2016), pp. 513-518.

${ }^{5}$ J. Bartl, F. Mühle, and L. Sætran, "Wind tunnel study on power output and yaw moments for two yaw-controlled model wind turbines," Wind Energy Sci. 3, 489-502 (2018).

${ }^{6}$ P. Fleming, J. Annoni, J. J. Shah, L. Wang, S. Ananthan, Z. Zhang, K. Hutchings, P. Wang, W. Chen, and L. Chen, "Field test of wake steering at an offshore wind farm," Wind Energy Sci. 2, 229-239 (2017).

${ }^{7}$ M. F. Howland, S. K. Lele, and J. O. Dabiri, "Wind farm power optimization through wake steering," Proc. Natl. Acad. Sci. U. S. A. 116, 14495-14500 (2019).

${ }^{8}$ P. Fleming, J. King, K. Dykes, E. Simley, J. Roadman, A. Scholbrock, P. Murphy, J. K. Lundquist, P. Moriarty, K. Fleming et al., "Initial results from a field campaign of wake steering applied at a commercial wind farm-Part 1," Wind Energy Sci. 4, 273 (2019).

${ }^{9}$ B. M. Doekemeijer, S. Kern, S. Maturu, S. Kanev, B. Salbert, J. Schreiber, F. Campagnolo, C. L. Bottasso, S. Schuler, F. Wilts et al., "Field experiment for open-loop yaw-based wake steering at a commercial onshore wind farm in Italy," Wind Energy Sci. (in review, 2020).

${ }^{10}$ I. Grant, P. Parkin, and X. Wang, "Optical vortex tracking studies of a horizontal axis wind turbine in yaw using laser-sheet, flow visualisation," Exp. Fluids 23, 513-519 (1997).

${ }^{11}$ J. Y. Liew, A. M. Urbán, and S. J. Andersen, "Analytical model for the poweryaw sensitivity of wind turbines operating in full wake," Wind Energy Sci. 5, 427-437 (2020).

${ }^{12}$ H. A. Madsen, N. N. Sørensen, and S. Schreck, "Yaw aerodynamics analyzed with three codes in comparison with experiment," in Wind Energy Symposium (2003), Vol. 75944, pp. 94-103.

${ }^{13} \mathrm{D}$. Medici, "Experimental studies of wind turbine wakes: power optimisation and meandering," Ph.D. thesis (KTH, 2005).

${ }^{14}$ J. Dahlberg and B. Montgomerie, "Research program of the utgrunden demonstration offshore wind farm, final report part 2, wake effects and other loads," Swedish Defense Research Agency, FOI, Kista, Sweden, Report No. FOI, 02-17, 2005.

${ }^{15}$ P. M. Gebraad, F. Teeuwisse, J.-W. van Wingerden, P. A. Fleming, S. D. Ruben, J. R. Marden, and L. Y. Pao, "A data-driven model for wind plant power optimization by yaw control,” in 2014 American Control Conference (IEEE, 2014), pp. 3128-3134.

${ }^{16}$ J. Jonkman, S. Butterfield, W. Musial, and G. Scott, "Definition of a 5-mw reference wind turbine for offshore system development," Technical Report No. NREL/TP-500-38060, National Renewable Energy Lab. (NREL), Golden, CO (United States), 2009.

${ }^{17}$ P.- $\AA$. Krogstad and M. S. Adaramola, "Performance and near wake measurements of a model horizontal axis wind turbine," Wind Energy 15, 743-756 (2012).

${ }^{18}$ J. Bartl, F. Mühle, J. Schottler, L. Sætran, J. Peinke, M. Adaramola, and M. Hölling, "Wind tunnel experiments on wind turbine wakes in yaw: Effects of inflow turbulence and shear," Wind Energy Sci. 3, 329 (2018).

${ }^{19}$ J. Schreiber, E. Nanos, F. Campagnolo, and C. L. Bottasso, "Verification and calibration of a reduced order wind farm model by wind tunnel experiments," J. Phys.: Conf. Ser. 854, 012041 (2017).

${ }^{20}$ M. Draper, A. Guggeri, B. López, A. Díaz, F. Campagnolo, and G. Usera, "A large eddy simulation framework to assess wind farm power maximization strategies: Validation of maximization by yawing," J. Phys.: Conf. Ser. 1037, 072051 (2018).

${ }^{21}$ H. Glauert, “Airplane propellers," in Aerodynamic Theory (Springer, 1935), pp. 169-360. 
${ }^{22}$ T. Burton, N. Jenkins, D. Sharpe, and E. Bossanyi, Wind Energy Handbook (John Wiley and Sons, 2011).

${ }^{23}$ H. A. Madsen, T. J. Larsen, G. R. Pirrung, A. Li, and F. Zahle, "Implementation of the blade element momentum model on a polar grid and its aeroelastic load impact," Wind Energy Sci. 5, 1-27 (2020).

${ }^{24}$ S. Boersma, B. Doekemeijer, P. M. Gebraad, P. A. Fleming, J. Annoni, A. K. Scholbrock, J. Frederik, and J.-W. van Wingerden, "A tutorial on controloriented modeling and control of wind farms," in 2017 American Control Conference (ACC) (IEEE, 2017).

${ }^{25}$ M. F. Howland, A. S. Ghate, S. K. Lele, and J. O. Dabiri, "Optimal closed-loop wake steering-Part 1: Conventionally neutral atmospheric boundary layer conditions," Wind Energy Sci. 5, 1315-1338 (2020).

${ }^{26} \mathrm{R}$. B. Stull, An Introduction to Boundary Layer Meteorology (Springer Science \& Business Media, 2012), Vol. 13.

${ }^{27}$ W. Miao, C. Li, J. Yang, and X. Xie, "Numerical investigation of the yawed wake and its effects on the downstream wind turbine," J. Renewable Sustainable Energy 8, 033303 (2016).

${ }^{28} \mathrm{C}$. L. Archer and A. Vasel-Be-Hagh, "Wake steering via yaw control in multiturbine wind farms: Recommendations based on large-eddy simulation," Sustainable Energy Technol. Assess. 33, 34-43 (2019).

${ }^{29}$ M. P. van der Laan and N. N. Sørensen, "Why the coriolis force turns a wind farm wake clockwise in the northern hemisphere," Wind Energy Sci. 2, 285 (2017).

${ }^{30} \mathrm{M}$. F. Howland, A. S. Ghate, and S. K. Lele, "Coriolis effects within and trailing a large finite wind farm," AIAA Paper No. 2020-0994, 2020.

${ }^{31}$ M. F. Howland, J. Bossuyt, L. A. Martínez-Tossas, J. Meyers, and C. Meneveau, "Wake structure in actuator disk models of wind turbines in yaw under uniform inflow conditions," J. Renewable Sustainable Energy 8, 043301 (2016).

${ }^{32} \mathrm{M}$. Bastankhah and F. Porté-Agel, "Experimental and theoretical study of wind turbine wakes in yawed conditions," J. Fluid Mech. 806, 506-541 (2016).

${ }^{33}$ L. A. Martínez-Tossas, J. Annoni, P. A. Fleming, and M. J. Churchfield, "The aerodynamics of the curled wake: A simplified model in view of flow control," Wind Energy Sci. 4, 127 (2019).

${ }^{34} \mathrm{H}$. Zong and F. Porté-Agel, "A point vortex transportation model for yawed wind turbine wakes," J. Fluid Mech. 890, A8 (2020).

${ }^{35}$ L. A. Martínez-Tossas, J. King, E. Quon, C. J. Bay, R. Mudafort, N. Hamilton, and P. Fleming, "The curled wake model: A three-dimensional and extremely fast steady-state wake solver for wind plant flows," Wind Energy Sci. Discuss. (in review, 2020).

${ }^{36} \mathrm{M}$. Abkar, J. N. Sørensen, and F. Porté-Agel, "An analytical model for the effect of vertical wind veer on wind turbine wakes," Energies 11, 1838 (2018).

${ }^{37}$ P. Fleming, A. Scholbrock, A. Jehu, S. Davoust, E. Osler, A. D. Wright, and A. Clifton, "Field-test results using a nacelle-mounted lidar for improving wind turbine power capture by reducing yaw misalignment," J. Phys.: Conf. Ser. 524, 012002 (2014).

${ }^{38} \mathrm{E}$. Hau, Wind Turbines: Fundamentals, Technologies, Application, Economics (Springer Science \& Business Media, 2013).

${ }^{39}$ M. A. Lackner, A. L. Rogers, and J. F. Manwell, "Uncertainty analysis in MCPbased wind resource assessment and energy production estimation," J. Solar Energy Eng. 130, 031006 (2008).

${ }^{40}$ C. Bay, J. R. King, P. A. Fleming, L. Martinez, R. M. Mudafort, E. J. Simley, and M. J. Lawson, FLORIS. Version 2.2.0 (2020). Available at https://github.com/ NREL/floris.

${ }^{41}$ C. R. Shapiro, D. F. Gayme, and C. Meneveau, "Modelling yawed wind turbine wakes: A lifting line approach," J. Fluid Mech. 841, R1 (2018).

${ }^{42} \mathrm{~J}$. C. Wyngaard, Turbulence in the Atmosphere (Cambridge University Press, 2010).

${ }^{43} \mathrm{~A}$. K. Blackadar, "The vertical distribution of wind and turbulent exchange in a neutral atmosphere," J. Geophys. Res. 67, 3095-3102, https://doi.org/10.1029/ JZ067i008p03095 (1962).

${ }^{44} \mathrm{~A}$. Constantin and R. Johnson, "Atmospheric Ekman flows with variable eddy viscosity," Boundary-Layer Meteorol. 170, 395-414 (2019).

${ }^{45} \mathrm{~A}$. K. Blackadar, "Boundary layer wind maxima and their significance for the growth of nocturnal inversions," Bull. Am. Meteorol. Soc. 38, 283-290 (1957).
${ }^{46} \mathrm{~A}$. Van Ulden and J. Wieringa, "Atmospheric boundary layer research at Cabauw," in Boundary-Layer Meteorology 25th Anniversary, edited by Garratt, J. R. and Taylor, P. A. (Springer, Dordrecht, 1996).

${ }^{47}$ M. F. Howland, A. S. Ghate, and S. K. Lele, "Influence of the horizontal component of earth's rotation on wind turbine wakes," J. Phys.: Conf. Ser. 1037, 072003 (2018)

${ }^{48}$ A. Englberger, J. K. Lundquist, and A. Dörnbrack, "Does the rotational direction of a wind turbine impact the wake in a stably stratified atmospheric boundary layer?," Wind Energy Sci. 5, 1359-1374 (2020).

${ }^{49} \mathrm{P}$. Murphy, J. K. Lundquist, and P. Fleming, "How wind speed shear and directional veer affect the power production of a megawatt-scale operational wind turbine," Wind Energy Sci. 5, 1169-1190 (2020).

${ }^{50} \mathrm{M}$. Sanchez Gomez and J. K. Lundquist, "The effect of wind direction shear on turbine performance in a wind farm in central Iowa," Wind Energy Sci. 5, 125 (2020).

${ }^{51}$ J. R. Taylor and S. Sarkar, "Stratification effects in a bottom Ekman layer," J. Phys. Oceanogr. 38, 2535-2555 (2008).

${ }^{52}$ M. F. Howland, A. S. Ghate, and S. K. Lele, "Influence of the geostrophic wind direction on the atmospheric boundary layer flow," J. Fluid Mech. 883, A39 (2020).

${ }^{53}$ E. Deusebio, G. Brethouwer, P. Schlatter, and E. Lindborg, "A numerical study of the unstratified and stratified ekman layer," J. Fluid Mech. 755, 672-704 (2014).

${ }^{54} \mathrm{~K}$. A. Kragh and M. H. Hansen, "Load alleviation of wind turbines by yaw misalignment," Wind Energy 17, 971-982 (2014).

${ }^{55} \mathrm{H}$. Glauert et al., A General Theory of the Autogyro (HM Stationery Office, 1926), Vol. 1111.

${ }^{56} \mathrm{G}$. Ingram, Wind Turbine Blade Analysis Using the Blade Element Momentum Method, Version 1.1 (Durham University, Durham, 2011).

${ }^{57}$ P. J. Moriarty and A. C. Hansen, “Aerodyn theory manual," Technical Report No. NREL/TP-500-36881, National Renewable Energy Lab., Golden, CO (US), 2005.

${ }^{58} \mathrm{M}$. Bastankhah and F. Porté-Agel, "Wind tunnel study of the wind turbine interaction with a boundary-layer flow: Upwind region, turbine performance, and wake region," Phys. Fluids 29, 065105 (2017).

${ }^{59} \mathrm{M}$. Bastankhah and F. Porté-Agel, "Wind farm power optimization via yaw angle control: A wind tunnel study," J. Renewable Sustainable Energy 11, 023301 (2019).

${ }^{60}$ E. Gaertner, J. Rinker, L. Sethuraman, F. Zahle, B. Anderson, G. E. Barter, N. J. Abbas, F. Meng, P. Bortolotti, W. Skrzypinski et al., "IEA wind TCP task 37: Definition of the IEA 15-megawatt offshore reference wind turbine," Technical Report No. NREL/TP-5000-75698, National Renewable Energy Lab. (NREL), Golden, CO (United States), 2020.

${ }^{61}$ J. C. Lagarias, J. A. Reeds, M. H. Wright, and P. E. Wright, "Convergence properties of the Nelder-Mead simplex method in low dimensions," SIAM J. Optim. 9, 112-147 (1998).

${ }^{62}$ C. Vera, J. Baez, M. Douglas, C. Emmanuel, J. Marengo, J. Meitin, M. Nicolini, J. Nogues-Paegle, J. Paegle, O. Penalba et al., "The south American low-level jet experiment," Bull. Am. Meteorol. Soc. 87, 63-78 (2006).

${ }^{63}$ S. Gadgil, "The Indian monsoon and its variability," Annu. Rev. Earth Planet. Sci. 31, 429-467 (2003).

${ }^{64} \mathrm{~L}$. Zhan, S. Letizia, and G. Valerio Iungo, "Lidar measurements for an onshore wind farm: Wake variability for different incoming wind speeds and atmospheric stability regimes," Wind Energy 23, 501-527 (2020).

${ }^{65}$ T. F. Pedersen, "On wind turbine power performance measurements at inclined airflow," Wind Energy 7, 163-176 (2004).

${ }^{66}$ P. Fleming, J. Annoni, M. Churchfield, L. A. Martinez-Tossas, K. Gruchalla, M. Lawson, and P. Moriarty, "A simulation study demonstrating the importance of large-scale trailing vortices in wake steering," Wind Energy Sci. 3, 243-255 (2018).

${ }^{67}$ M. A. Lackner, A. L. Rogers, J. F. Manwell, and J. G. McGowan, "A new method for improved hub height mean wind speed estimates using short-term hub height data," Renewable Energy 35, 2340-2347 (2010).

${ }^{68}$ R. Damiani, S. Dana, J. Annoni, P. Fleming, J. Roadman, J. van Dam, and K. Dykes, "Assessment of wind turbine component loads under yaw-offset conditions," Wind Energy Sci. 3, 173-189 (2018). 